\title{
Frühkindliche Karies und assoziierte Risikofaktoren bei Kleinkindern im Land Brandenburg
}

Dissertation zur Erlangung des akademischen Grades doctor medicinae dentariae (Dr. med. dent.)

vorgelegt dem Rat der Medizinischen Fakultät der Friedrich-Schiller-Universität Jena

von Martin Deichsel

geboren am 13.06.1987 in Brandenburg an der Havel 
Gutachter:

1) Prof. Dr. Roswitha Heinrich-Weltzien, Jena

2) Prof. Dr. Dr. Bernd W. Sigusch, Jena

3) Prof. Dr. Ulrich Schiffner, Hamburg

Tag der öffentlichen Verteidigung: $\quad$ 04. Februar 2014 


\section{Abkürzungsverzeichnis}

$\begin{array}{ll}\text { AAPD } & \text { American Academy of Pediatric Dentistry } \\ \text { BSI } & \text { Brandenburger Sozialindex } \\ \text { dmfs } & \text { decayed, missing, filled-surface Index } \\ \text { dmft } & \text { decayed, missing, filled-tooth Index } \\ \text { EAPD } & \text { European Academy of Paediatric Dentistry } \\ \text { ECC } & \text { Early Childhood Caries } \\ \text { FDI } & \text { World Dental Federation (Fédération Dentaire International) } \\ \text { GB } & \text { Großbritannien } \\ \text { HE } & \text { Hessen } \\ \text { Kita } & \text { Kindertagesstätte } \\ \text { MV } & \text { Mecklenburg-Vorpommern } \\ \text { NI } & \text { Niedersachsen } \\ \text { NW } & \text { Nordrhein-Westfalen } \\ \text { ÖGD } & \text { Öffentlicher Gesundheitsdienst } \\ \text { OR } & \text { Odds Ratio } \\ \text { S-ECC } & \text { Severe-Early Childhood Caries } \\ \text { SH } & \text { Schleswig-Holstein } \\ \text { SiC } & \text { Significant Caries Index } \\ \text { SN } & \text { Sachsen } \\ \text { SPSS } & \text { Statistik und Analysesoftware, SPSS Statistics } \\ \text { TH } & \text { Thüringen } \\ \text { WHO } & \text { World Health Organization } \\ \text { ZÄD } & \text { Zahnärztlicher Dienst } \\ & \end{array}$


Inhaltsverzeichnis

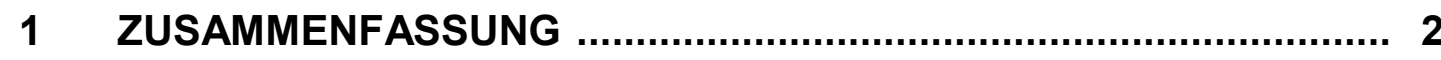

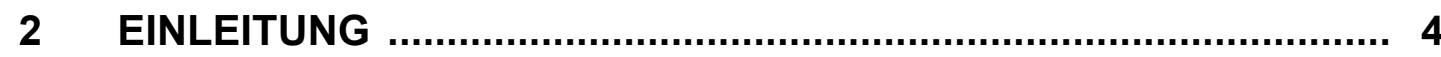

2.1 Definition und Ätiologie der frühkindlichen Karies ……............. 4

2.1.1 Risikofaktoren der frühkindlichen Karies …........................... 7

2.2 Epidemiologie der frühkindlichen Karies ………..................... 9

2.2.1 Prävalenz der frühkindlichen Karies in Deutschland ................. 12

2.3 Prävention der frühkindlichen Karies ...................................... 13

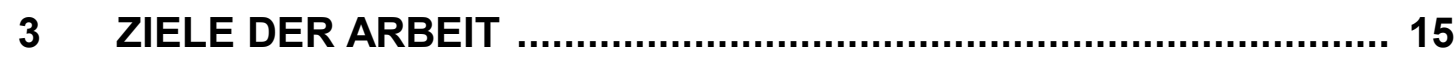

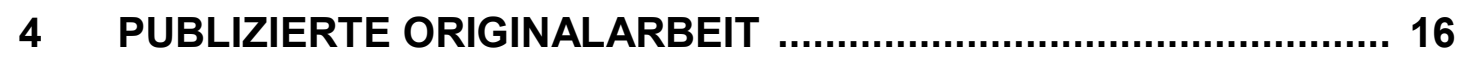

Frühkindliche Karies und assoziierte Risikofaktoren bei Kleinkindern im Land Brandenburg.

Deichsel M, Rojas G, Lüdecke K, Heinrich-Weltzien R.

Bundesgesundheitsblatt 55 (11-12): 1504-1511, 2012

5 WEITERFÜHRENDE UNTERSUCHUNGEN UND ERGEBNISSE .... 25

5.1 Kariesprävalenz und -befall in den einzelnen Kreisen im Land Brandenburg

5.2 Einfluss des Sozialstatus auf den Kariesbefall und das Mundgesundheitsverhalten bei Kleinkindern im Land Brandenburg

5.3 Ergebnisse der Elternbefragung zum Mundgesundheitsverhalten bei ihren Kindern

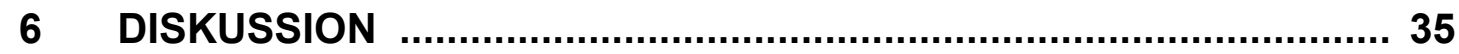

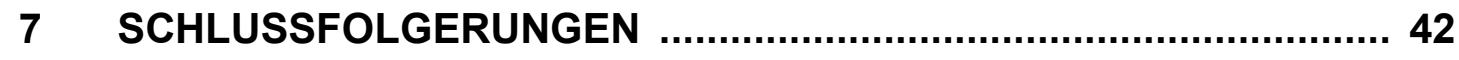

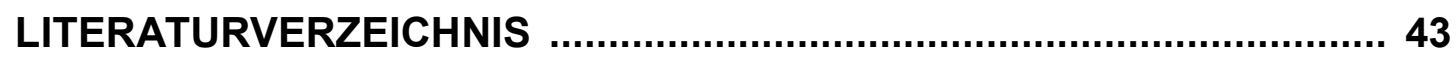

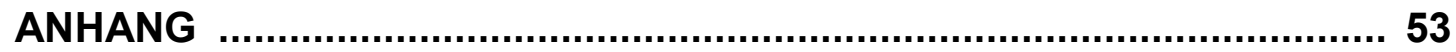




\section{Zusammenfassung}

Hintergrund und Zielstellung: Die frühkindliche Karies (Early Childhood Caries, ECC) ist eine Erkrankung von unter 71 Monate alten Kindern, bei denen mindestens ein Zahn eine Karies aufweist oder gefüllt, beziehungsweise extrahiert wurde. Die ECC ist weltweit die häufigste chronische Erkrankung bei Kleinkindern. Folgen der ECC für das Kleinkind können Schmerzen, eine Keimschädigung der nachfolgenden permanenten Zähne oder ein Platzmangel im bleibenden Gebiss sein. Darüber hinaus kann es zu Sprachstörungen und einem negativen Einfluss auf das schulische Leistungsvermögen und die soziale Kompetenz kommen. Neben einer Infektanfälligkeit der Kinder wurde in jüngsten Untersuchungen eine Assoziation zwischen schweren Formen der ECC und einer Eisenmangelanämie nachgewiesen. Aufgrund der fehlenden Compliance der Kleinkinder ist die Sanierung des Milchgebisses vielfach nur in Intubationsnarkose durchführbar; sie ist mit einem hohen Kostenaufwand für das Gesundheitssystem verbunden.

Ziel der Untersuchung war es, die Kariesprävalenz und den Kariesbefall sowie deren Abhängigkeit vom Gesundheitsverhalten und dem Sozialstatus der Eltern bei Kindern unter 3 Jahren im Land Brandenburg zu ermitteln und Risikofaktoren aufzudecken. Die Ergebnisse der Untersuchung sollen zur kritischen Bewertung bestehender Präventionsprogramme herangezogen werden und/oder in Planung befindliche Programme konzeptionell unterstützen.

Methoden: Es wurde der Kariesbefall von 661 Kindern, die in Kindertagesstätten (Kitas) und von Tagesmüttern/-vätern in 10 Landkreisen und kreisfreien Städten betreut wurden, nach WHO Standard (1997) durch kalibrierte Zahnärztinnen des Öffentlichen Gesundheitsdienstes (ÖGD) erfasst und das Gesundheitsverhalten sowie der Sozialstatus der Eltern (Brandenburger Sozialindex) fragebogenbasiert ermittelt. Es erfolgte eine anonymisierte Verknüpfung der Daten. Zusammenhänge zwischen Fragebogenvariablen und Kariesbefall wurden zunächst bivariat mit dem Fisher's exakt Test geprüft. Signifikante Variablen wurden nach einer Alters- und Geschlechtsadjustierung des Modells schrittweise in einer multiplen, binären logistischen Regressionsanalyse erneut auf ihre Signifikanz untersucht.

Ergebnisse: Die Kariesprävalenz lag in der Stichprobe der 13-36 Monate alten Kinder auf Schmelzkaries-(d1-4)-Niveau bei $7,4 \%$ und auf Dentinkaries-(d3-4)- 
Niveau bei $5,3 \%$, wobei $25-36$ Monate alte Kinder signifikant häufiger Karies aufwiesen als 13-24 Monate alte. Der Kariesbefall betrug 0,3 ( \pm 1,2) d1-4mft bzw. 0,2 $( \pm 1,0)$ d3-4mft. Da keine signifikanten Unterschiede bei Kariesprävalenz, Kariesbefall sowie der Alters- und Geschlechtsverteilung in der Stichprobe mit Elternbefragung ( $n=661)$ und der Gesamtpopulation aller Kinder in den 10 Kreisen ( $n=10.161)$ festgestellt wurden, kann die Stichprobe als repräsentativ betrachtet werden. Der SiC-Index, der das Drittel einer Population mit dem höchsten Kariesbefall charakterisiert, lag bei 0,8 $( \pm 2,0)$ d1-4mft. 2\% der Kinder vereinten $52 \%$ des Kariesbefalls auf sich. Der Sanierungsgrad betrug 19,9\% und der Füllungsindex $10 \%$.

Die Auswertung der bivariaten Analyse ergab, dass Kinder mit Karies signifikant länger die Saugerflasche verabreicht bekamen, häufiger nächtlichen Zugang zur Flasche hatten, ihre Eltern öfter jünger als 20 Jahre waren, häufiger mit allein erziehenden Elternteilen aufwuchsen, zu einem signifikant größeren Anteil einen niedrigen Sozialstatus aufwiesen, häufiger erst nach dem 2. Lebensjahr beim Zahnarzt waren und ihre Eltern öfter keine zahnärztliche Aufklärung zur ECC erhielten. Die multivariate logistische Regressionsanalyse zeigte, dass der Sozialstatus, das Alter des Kindes und die nächtliche Saugerflaschengabe die Hauptrisikofaktoren der ECC sind. So liegt das Risiko eines Kindes mit niedrigem Sozialstatus 7,34-fach höher (OR) an Karies zu erkranken, als das eines Kindes mit hohem Sozialstatus. Das Risiko für 25-36 Monate alte Kinder und Kinder mit nächtlichem Zugang zur Saugerflasche war 3,84- bzw. 2,4-fach höher (OR) als das Jüngerer und von Kindern ohne nächtliche Flaschengabe.

Schlussfolgerungen: In dieser Studie wurden erstmalig landesweite Daten zur ECC-Prävalenz und ihrer Assoziation zum Gesundheitsverhalten und Sozialstatus der Eltern erhoben. Gruppenprophylaktische Maßnahmen in Kitas sind nur dann erfolgreich, wenn sie auf die Risikofaktoren der ECC abzielen. Dies sollte durch flächendeckende, setting-orientierte Präventionsprogramme, die das tägliche Zähneputzen in den Kitas mit fluoridhaltiger Zahnpasta und den Verzicht auf die Saugerflasche fördern, sowie durch eine begleitende Gesundheitsförderung mit Hebammen-, Schwangeren-, und Elternschulungen möglich sein. Gleichzeitig ist eine stärkere Zusammenarbeit zwischen Zahnärzten des ÖGD, niedergelassenen Zahnärzten und Pädiatern sowie Kitas und Eltern erforderlich, um die gesundheitliche Benachteiligung von Kindern mit niedrigem Sozialstatus zu kompensieren. 


\section{$2 \quad$ Einleitung}

\subsection{Definition und Ätiologie der frühkindlichen Karies}

Um das gesunde Aufwachsen eines Kindes zu ermöglichen, muss auch Wert auf die Mundgesundheit der Jüngsten gelegt werden. Orale Erkrankungen im frühen Kindesalter können schwerwiegende Auswirkungen auf die spätere Entwicklung haben. Trotz aller präventiven Anstrengungen ist die frühkindliche Karies immer noch die häufigste chronische Erkrankung bei Kleinkindern (PHSCC 2000, FDI 2012). Als frühkindliche Karies wird das Vorliegen mindestens einer kariösen (sowohl kavitierten als auch nicht-kavitierten) Läsion, das Fehlen von Zähnen auf Grund von Karies oder das Vorhandensein von einer oder mehreren Füllungen, bei Kindern unter 6 Jahren, bezeichnet (AAPD 2011).

Die frühkindliche Karies wurde erstmals Mitte des 19. Jahrhunderts durch den deutsch-amerikanischen Kinderarzt Abraham Jacobi (1862) beschrieben. 100 Jahre später nannte der Kinderzahnarzt Elias Fass das Krankheitsbild „nursing bottle mouth“ (1962). Später fanden Begriffe wie „nursing caries“, „baby bottle caries“ oder „baby bottle tooth decay" beziehungsweise "Flaschenkaries" oder „Babyflaschenkaries“ in der Literatur Verwendung (Horowitz 1998). Da diese Bezeichnungen jedoch implizieren, dass nur das Stillen oder die Flaschengabe allein Ursache für die Kariesentstehung bei Klein- und Kleinstkindern ist und die multifaktorielle Ätiologie der Karies außer Acht gelassen wurde, empfahl das USamerikanische "Center for Disease Control and Prevention" im Jahr 1994 eine Vereinheitlichung der Terminologie auf den Begriff "Early Childhood Caries“ (ECC) (Kaste und Gift 1995). Die American Academy of Pediatric Dentistry (AAPD) definiert darüber hinaus auch das Krankheitsbild einer schweren Form der ECC „Severe-Early Childhood Caries" (S-ECC) (AAPD 2011). Diese liegt vor, wenn bei Kindern unter 3 Jahren eine Karies an den Glattflächen der Zähne diagnostiziert werden kann oder wenn bei Kindern zwischen 3 und 5 Jahren mindestens eine kavitierte kariöse Läsion, auf Grund von Karies fehlende oder gefüllte Glattfläche, der Oberkieferfrontzähne vorliegt. Man spricht ebenso von einer schweren frühkindlichen Karies, wenn die Summe der kariösen, fehlenden oder gefüllten Glattflächen der Zähne bei 3-Jährigen größer/gleich 4, bei 4-Jährigen größer/gleich 5 oder bei 5- 
Jährigen größer/gleich 6 ist (AAPD 2011). Eine inzwischen im klinischen Altag etablierte Klassifikation der ECC wurde von Wyne (1999) vorgenommen (Tab. 1).

Tab. 1: Klassifikation der frühkindlichen Karies nach Wyne (1999)

\begin{tabular}{|c|l|l|}
\hline ECC Typ & \multicolumn{1}{|c|}{ Klassifikation } & \multicolumn{1}{|c|}{ Erscheinungsbild } \\
\hline ECC Typ 1 & $\begin{array}{l}\text { leichte bis mittelschwere } \\
\text { ECC }\end{array}$ & $\begin{array}{l}\text { Einzelne kariöse Läsionen an Molaren } \\
\text { und/ oder Inzisiven }\end{array}$ \\
\hline ECC Typ 2 & $\begin{array}{l}\text { mittelschwere bis schwere } \\
\text { ECC }\end{array}$ & $\begin{array}{l}\text { Kariöse Läsionen an den } \\
\text { Oberkieferinzisiven mit oder ohne } \\
\text { Beteiligung der Molaren }\end{array}$ \\
\hline ECC Typ 3 & schwere ECC & $\begin{array}{l}\text { Kariöse Läsionen an fast allen } \\
\text { Milchzähnen inklusive der unteren } \\
\text { Inzisiven }\end{array}$ \\
\hline
\end{tabular}

Die Diagnostik der frühkindlichen Karies kann meist rein visuell erfolgen. Initialkariöse Läsionen stellen sich als weißlich-opake Verfärbungen (sogenannte „white-spot lesions") dar, die anfangs meist girlandenförmig, der Neonatallinie des Zahnes folgend, an den Glattflächen der Oberkieferfrontzähne verlaufen. Im weiteren Verlauf kommt es dann zur Kavitation der Läsionen, die bis zur vollständigen Zerstörung der Zahnkrone führen kann. Das für die frühkindliche Karies typische Befallsmuster folgt dabei der Sequenz des Zahndurchbruchs und ist durch das Umspülen der Oberkieferfrontzähne mit erosiven und/oder kariogenen Flüssigkeiten beim Trinken aus der Saugerflasche bedingt (De Grauwe et al. 2004).

Karies an sich ist eine multifaktorielle, chronische Erkrankung der Zahnhartsubstanz, die hauptsächlich durch Mutans-Streptokokken ausgelöst wird. Die Verstoffwechselung niedermolekularer Kohlenhydrate erzeugt dabei organische Säuren, wobei durch die Herabsetzung des $\mathrm{pH}-$ Wertes, Mineralien aus den Zahnhartgeweben herausgelöst werden (Loesche 1986). Wenn die Zahnhartgewebe durch den Mangel an Mineralien in ihrer Struktur geschwächt sind, kommt es zur Kavitation der kariösen Läsion. Voraussetzung für das Entstehen einer frühkindlichen Karies sind neben dem Vorhandensein eines Wirtes (ab dem Zahndurchbruch im ca. 6. Monat), die Infektion mit Streptococcus mutans, eine Plaqueansammlung auf der Zahnoberfläche, regelmäßige Substratzufuhr in Form niedermolekularer Kohlenhydrate und ausreichend Zeit für die Demineralisationsprozesse (Abb. 1) (Gussy et al. 2006). Da der Zahnschmelz der Milchzähne bei ihrem Durchbruch noch nicht ausgereift und mit 
Speichelionen, insbesondere Fluoridionen, gesättigt ist, besteht eine erhöhte Kariesanfälligkeit (Simmer und $\mathrm{Hu}$ 2001). Die Infektion mit Streptococcus mutans erfolgt in der Regel nach Durchbruch der ersten Milchzähne durch den elterlichen Speichel (Wan et al. 2001); jedoch konnte auch bei zahnlosen Säuglingen eine perinatale, vaginal-orale Infektion festgestellt werden ( $\mathrm{Li}$ et al. 2005), wobei die Bakterien bis zur Anheftung an der Zahnoberfläche in gingivalen Fibroblasten überleben (Berlutti et al. 2010). Die Bildung der dentalen Plaque vollzieht sich innerhalb kurzer Zeit durch die Anheftung von Bakterien an der aus Speichelproteinen bestehenden Pellikelschicht des Zahnes. Dabei kommt es zur Ausbildung eines mikrobiellen, wasserunlöslichen Biofilms und zur Ansiedlung weiterer, zunehmend anaerober und säureresistenter Mikroorganismen (Marsh et al. 2011).

Das wichtigste Substrat für die Kariesentstehung ist der Zucker Saccharose. Neben organischen Säuren entstehen bei der Verstoffwechselung der Saccharose auch Dextrane, die zur Bildung und Stabilisierung der Plaque wesentlich beitragen (Mikkelsen 1996). Entscheidend bei der Kariesentstehung ist jedoch nicht die Menge des zugeführten Substrates, sondern die Häufigkeit und Frequenz der Aufnahme von niedermolekularen Kohlenhydraten, da der orale $\mathrm{pH}-$ Wert sich durch den Speichelfluss, bei hoch frequenter Zufuhr, nicht wieder normalisieren kann und somit keine Remineralisation der Zahnhartsubstanzen möglich ist (Milgrom et al. 2000).

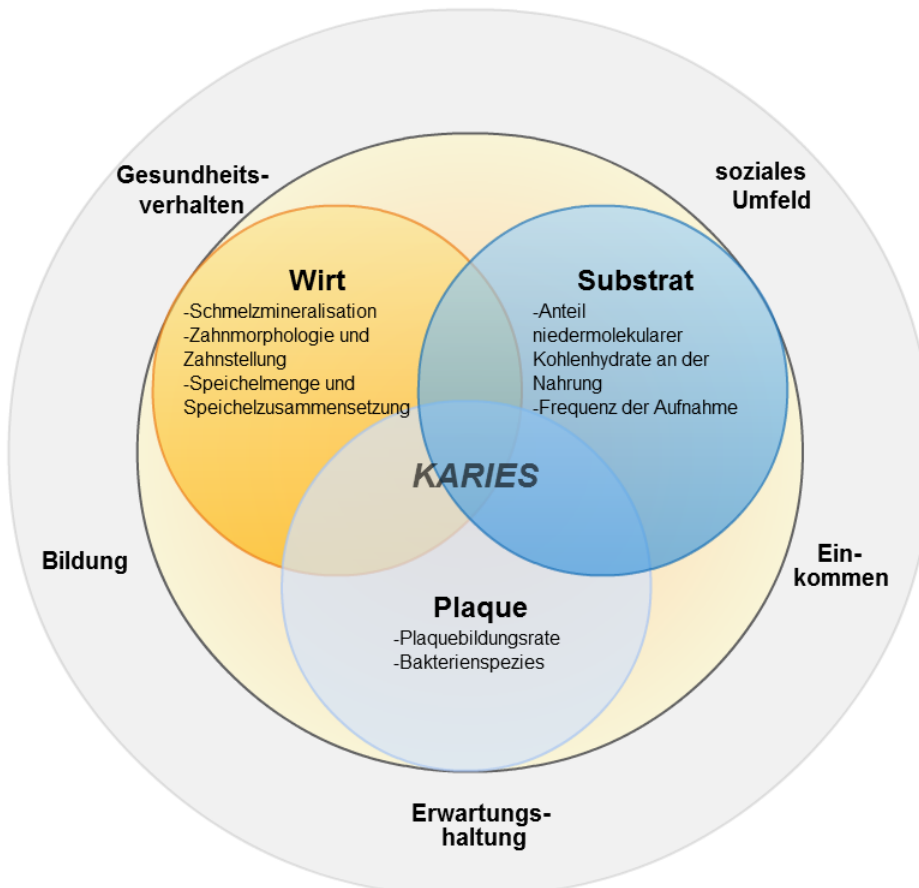

Abb. 1: Kariesätiologie (modifiziert nach Hellwig et al. (2009)) 
Die Folgen der frühkindlichen Karies sind vielfältig und können sich weit über die Gebrauchsperiode der Milchzähne hinaus auswirken. Direkte Folgen der ECC sind Schmerzen und damit verbundene Einschränkungen der Nahrungsaufnahme sowie aus nicht behandelter Karies resultierende Abszedierungen, die mit einer Schädigung der Zahnkeime der bleibenden Dentition einhergehen können (Wetzel 2008). Die erforderliche Extraktion der stark zerstörten Milchzähne hat vielfach Auswirkungen auf das Platzangebot im bleibenden Gebiss. Mit dem vorzeitigen Milchzahnverlust assoziierte Engstandsymptome erfordern in der Regel eine spätere kieferorthopädische Behandlung (Robke 2008). Weiterhin kann die Sprachentwicklung des Kindes durch die erschwerte Lautbildung beim Fehlen eines oder mehrerer Zähne beeinträchtigt werden. Dies kann zum Sigmatismus führen und auch negative psychische Konsequenzen für das Kind haben (Abanto et al. 2011). Das schulische Leistungsvermögen und die soziale Kompetenz können als Folge der ECC beeinträchtigt sein (Blumenshine et al. 2008), ebenso sinkt die Lebensqualität des Kindes allgemein (Leal et al. 2012). Die Infektanfälligkeit der Kinder ist erhöht und die oftmals nur in Intubationsnarkose durchführbare Sanierung der Milchgebisse ist mit einem erhöhten Risiko für das Kind und einem hohen Kostenaufwand für das Gesundheitssystem verbunden (Casamassimo et al. 2009). Nach neuen Erkenntnissen kann die ECC auch zur Ausbildung einer Eisenmangelanämie im Kindesalter führen, was vermutlich auf das chronische Entzündungsgeschehen bei einer Pulpenbeteiligung oder Abszessbildung zurückzuführen ist (Shaoul et al. 2012, Schroth et al. 2013). Untersuchungen zeigen darüber hinaus, dass das Kariesrisiko im Erwachsenenalter in Relation zur ECC-Erfahrung des Kindes steht (al-Shalan et al. 1997).

\subsubsection{Risikofaktoren der frühkindlichen Karies}

Die Risikofaktoren für die Erkrankung an einer frühkindlichen Karies sind vielfältig. Neben einer inadäquaten Mundhygiene und kariogenen Ernährungsgewohnheiten, spielen sozioökonomische Faktoren eine wesentliche Rolle.

Schon die frühzeitige Infektion der Mundhöhle des Kindes mit Streptococcus mutans stellt einen erhöhtes Risiko für die ECC dar. Dabei ist die Konzentration von Mutansstreptokokken im Speichel der Mutter von Bedeutung, da die Bakterien hauptsächlich vertikal durch den Speichel der Eltern, beim Küssen, Verwenden des gleichen Löffels oder ablutschen des Nuckels übertragen werden (De Grauwe et al. 
2004). Da eine hohe Konzentration von Streptococcus mutans im Mund der Mutter die Wahrscheinlichkeit für eine frühzeitige Keimübertragung erhöht, stellt auch eine insuffiziente elterliche Mundhygiene ein erhöhtes ECC-Risiko für das Kind dar (Tinanoff et al. 2002). Der Verzicht auf 2-mal tägliches Zähneputzen mit fluoridhaltiger Kinderzahnpasta ab dem Durchbruch des ersten Milchzahnes ist ebenso ein Risikofaktor, da die Plaquebildung nicht unterbunden wird und bei einer zu geringen Fluoridzufuhr die posteruptive Mineralisation der Milchzähne unterbleibt (Tsai et al. 2006, Declerck et al. 2008, Splieth et al. 2009, Hellwig et al. 2010, Subramaniam und Prashanth 2012). Schmelzhypoplasien an den Milchzähnen sind ebenfalls Risikofaktoren für eine ECC (Gussy et al. 2006, Zhou et al. 2012). Strukturstörungen des Zahnschmelzes sind jüngsten Untersuchungen zu Folge vielfach mit einer schweren Form der ECC (hypoplasia-associated severe early childhood caries, HAS-ECC) assoziiert (Caufield et al. 2012).

Ein entscheidender Risikofaktor ist die falsche Ernährung der Kleinkinder. Hierbei ist vor allem der Konsum zuckerreicher Zwischenmahlzeiten und die Gabe erosiver und kariogener Getränke, wie Fruchtsäfte, zuckerhaltiger Milchgetränke oder Softdrinks, mit der Saugerflasche bedeutsam (Declerck et al. 2008, Qin et al. 2008). Besonders gravierend sind die Folgen der frequenten Gabe der Saugerflasche in der Nacht, da der Speichelfluss mit seiner neutralisierenden Wirkung, in der Nacht herabgesetzt ist (Berkowitz 2003). Die oftmals säurehaltigen Getränke fördern und beschleunigen durch die erosive Schädigung des Zahnschmelzes den kariogenen Prozess. Zudem werden die Oberkieferfrontzähne durch den Saugerflaschenaufsatz permanent umspült (Lussi et al. 2000). Auch erosive oder zuckerhaltige Medikamente, wie beispielsweise Inhalatoren oder Hustensäfte können bei häufigem Gebrauch zur Kariesentstehung führen (Horowitz 1998). Ob es einen Zusammenhang zwischen dem Stillen des Kindes und einem erhöhten ECC-Risiko gibt wird kontrovers diskutiert. Einige Autoren vetreten die Auffassung, dass Stillen per se kein Risikofaktor für die ECC-Entstehung darstellt (Hallett und O'Rourke 2003, Gussy et al. 2006, Mohebbi et al. 2008). Weiterhin empfiehlt die WHO ebenso wie das britische Department of Health das Stillen von Kleinkindern als gesündeste Ernährungsweise (WHO 2003, BASCD 2009). Demgegenüber stellen einige Autoren heraus, dass Muttermilch durch ihren höheren Laktoseanteil (7,4\% gegenüber 4,8\% in Kuhmilch) einen kariesfördernden Einfluss haben könnte, wenn das Stillen sehr 
häufig, in der Nacht oder öfter als 2-mal täglich nach dem ersten Lebensjahr erfolgt (De Grauwe et al. 2004, Azevedo et al. 2005).

Auch das Umfeld in dem das Kind aufwächst, also sein sozioökonomischer Hintergrund, wurde bezüglich des Risikopotentials für eine ECC vielfach untersucht. So erkranken Kinder die in Haushalten mit einem allein erziehenden Elternteil aufwachsen häufiger an ECC als Kinder die mit beiden Elternteilen im Haushalt leben (Milnes 1996, Hallett und O'Rourke 2003, De Grauwe et al. 2004). Auch ein junges Alter der Mutter bei Geburt des Kindes kann ein erhöhtes Kariesrisiko für das Kleinkind darstellen (Hallett und O'Rourke 2003, Niji et al. 2010). Ebenso wird in einigen Studien berichtet, dass die Kariesprävalenz mit der Anzahl der Geschwister proportional ansteigt (Schroth und Cheba 2007). Weiterhin stellt ein Migrationshintergrund der Familie einen bedeutenden Risikofaktor dar (Micheelis et al. 2007, Pieper und Jablonski-Momeni 2008). Andere sozioökonomische Faktoren, die einen Einfluss auf das Kariesrisiko des Kindes haben, sind eine niedrige Schulbildung der Eltern, insbesondere der Mutter (Psoter et al. 2006, Finlayson et al. 2007, Qin et al. 2008, Zhou et al. 2012) und ein geringes Einkommen oder Arbeitslosigkeit in der Familie (Gussy et al. 2006, Psoter et al. 2006, Finlayson et al. 2007, Declerck et al. 2008, Feldens et al. 2010, Subramaniam und Prashanth 2012), die als niedriger sozioökonomischer- oder Sozialstatus zusammengefasst werden können.

\subsection{Epidemiologie der frühkindlichen Karies}

Die Prävalenz der ECC ist noch nicht flächendeckend, weltweit untersucht worden. Der Vergleich einzelner Studien ist durch die unterschiedlich angewandten Diagnosekriterien, die Betrachtung verschiedener Altersgruppen und die teilweise auf Risikogruppen begrenzte Stichprobenauswahl erschwert. Der Kariesbefall im Milchgebiss folgt weltweit bislang nicht dem Trend eines "caries decline", der in den Industrienationen seit den 70er Jahren auf Grund der weiten Verfügbarkeit von Fluoriden beobachtet wurde (Marthaler et al. 1996, Stecksen-Blicks et al. 2004). Die ECC ist ein globales Problem und wurde daher in einem jüngsten Positionspapier der World Dental Federation (FDI) als eine Erkrankung charakterisiert, die es mit intersektoralen Präventionskonzepten zu bekämpfen gilt (Fisher et al. 2012). In jedem Land ist die Zahnärzteschaft und ihre Standespolitik aufgefordert, evidenzbasierte Programme zu entwickeln. Weiterhin zeichnet sich eine zunehmende 
Polarisation des Kariesbefalls auf sozial benachteiligte Gruppen ab (Carvalho et al. 2004).

Gegenwärtig wird weltweit eine Prävalenz der ECC zwischen 3 und 83\% beobachtet (Tab. 2), wobei die Prävalenz in den Industrienationen am niedrigsten und in den Entwicklungsländern am höchsten ist (Ismail und Sohn 1999). Innerhalb eines Landes zeigt sich eine höhere Prävalenz in sozial deprivierten Gruppen und Minoritäten einer Gesellschaft (Psoter et al. 2006).

Tab. 2: Übersicht zur weltweiten Prävalenz der ECC

\begin{tabular}{|c|c|c|c|c|}
\hline Studie & Stichprobenauswahl & Region & $\begin{array}{c}\text { Kariesprävalenz } \\
(\%)\end{array}$ & $\begin{array}{l}\text { Kariesbefall } \\
\text { (dmft) }\end{array}$ \\
\hline $\begin{array}{l}\text { Abanto et al. } \\
\text { (2011) }\end{array}$ & $\begin{array}{l}\text { 2-5-Jährige } \\
(n=260)\end{array}$ & $\begin{array}{l}\text { Sao Paulo, } \\
\text { Brasilien }\end{array}$ & 63,8 & 7,3 \\
\hline $\begin{array}{l}\text { Al-Malik et al. } \\
(2001)\end{array}$ & $\begin{array}{l}\text { 2-5-Jährige } \\
(n=987)\end{array}$ & $\begin{array}{l}\text { Jeddah, } \\
\text { Saudi- } \\
\text { Arabien }\end{array}$ & 73,0 & k.A. \\
\hline $\begin{array}{l}\text { Azevedo et al. } \\
\text { (2005) }\end{array}$ & $\begin{array}{l}\text { 36-71-Monatige } \\
(n=369)\end{array}$ & Brasilien & 36,0 & k.A. \\
\hline $\begin{array}{l}\text { Declerck et al. } \\
(2008)\end{array}$ & $\begin{array}{l}\text { 3-Jährige } \\
(n=1250)\end{array}$ & $\begin{array}{l}\text { Flandern, } \\
\text { Belgien }\end{array}$ & 6,9 & k.A. \\
\hline Dye et al. (2007) & 2-5-Jährige & USA & 27,9 & 1,2 \\
\hline $\begin{array}{l}\text { Feldens et al. } \\
(2010)\end{array}$ & $\begin{array}{l}\text { 4-Jährige } \\
(n=340)\end{array}$ & $\begin{array}{l}\text { Sao } \\
\text { Leopoldo, } \\
\text { Brasilien }\end{array}$ & 63,0 & 3,8 \\
\hline $\begin{array}{l}\text { Ferro et al. } \\
(2004)\end{array}$ & $\begin{array}{l}\text { 0-36-Monatige } \\
(\mathrm{n}=29)\end{array}$ & Italien & 17,2 & k.A. \\
\hline $\begin{array}{l}\text { Haugejorden und } \\
\text { Birkeland (2002) }\end{array}$ & 5-Jährige & Norwegen & 38,9 & 1,5 \\
\hline Horowitz (1998) & 1-3-Jährige & USA & $3-6$ & k.A. \\
\hline $\begin{array}{l}\text { Kalyvas et al. } \\
(2006)\end{array}$ & $\begin{array}{l}\text { 5-Jährige } \\
(n=345)\end{array}$ & $\begin{array}{l}\text { Attika, } \\
\text { Griechenland }\end{array}$ & 48,4 & 2,6 \\
\hline $\begin{array}{l}\text { Kumarihamy et } \\
\text { al. (2011) }\end{array}$ & $\begin{array}{l}\text { 1-2-Jährige } \\
(n=410)\end{array}$ & $\begin{array}{l}\text { Colombo, } \\
\text { Sri Lanka }\end{array}$ & 32,2 & 2,0 \\
\hline $\begin{array}{l}\text { Livny et al. } \\
(2007)\end{array}$ & $\begin{array}{l}\text { 30-Monatige } \\
(n=596)\end{array}$ & $\begin{array}{l}\text { Jerusalem, } \\
\text { Israel }\end{array}$ & 15,3 & k.A. \\
\hline
\end{tabular}




\begin{tabular}{|c|c|c|c|c|}
\hline $\begin{array}{l}\text { Lulic-Dukic et al. } \\
\text { (2001) }\end{array}$ & $\begin{array}{l}\text { 2-5-Jährige } \\
(n=145)\end{array}$ & Kroatien & 30,0 & k.A. \\
\hline $\begin{array}{l}\text { Mohebbi et al. } \\
(2008)\end{array}$ & $\begin{array}{l}\text { 1-3-Jährige } \\
(n=504)\end{array}$ & $\begin{array}{l}\text { Teheran, } \\
\text { Iran }\end{array}$ & $3-26$ & k.A. \\
\hline Pitts et al. (2005) & 5-Jährige & $\begin{array}{l}\text { Schottland, } \\
\text { GB }\end{array}$ & 55,4 & 2,8 \\
\hline Pitts et al. (2005) & 5-Jährige & $\begin{array}{l}\text { England und } \\
\text { Wales, GB }\end{array}$ & 39,6 & 1,6 \\
\hline $\begin{array}{l}\text { Poulsen und } \\
\text { Malling Pedersen } \\
\text { (2002) }\end{array}$ & 5-Jährige & Dänemark & 29,0 & 1,0 \\
\hline $\begin{array}{l}\text { Rosenblatt und } \\
\text { Zarzar (2002) }\end{array}$ & $\begin{array}{l}\text { 12-36-Monatige } \\
(\mathrm{n}=468)\end{array}$ & $\begin{array}{l}\text { Recife, } \\
\text { Brasilien }\end{array}$ & 28,5 & k.A. \\
\hline $\begin{array}{l}\text { Santos und } \\
\text { Soviero (2002) }\end{array}$ & $\begin{array}{l}\text { 0-36-Monatige } \\
(n=77)\end{array}$ & $\begin{array}{l}\text { Rio de } \\
\text { Janeiro, } \\
\text { Brasilien }\end{array}$ & 41,6 & k.A. \\
\hline $\begin{array}{l}\text { Sufia et al. } \\
\text { (2009) }\end{array}$ & $\begin{array}{l}\text { 0-5-Jährige } \\
(n=600)\end{array}$ & $\begin{array}{l}\text { Lahore, } \\
\text { Pakistan }\end{array}$ & 40,8 & k.A. \\
\hline Tsai et al. (2006) & $\begin{array}{l}\text { 0-3-Jährige } \\
(n=478)\end{array}$ & Taiwan & 24,7 & k.A. \\
\hline $\begin{array}{l}\text { Vachirarojpisan } \\
\text { et al. (2004) }\end{array}$ & 15-19-Monatige & Thailand & 82.8 & 4,2 \\
\hline $\begin{array}{l}\text { Warren et al. } \\
(2008)\end{array}$ & $\begin{array}{l}\text { 6-24-Monatige } \\
(\mathrm{n}=187)\end{array}$ & $\begin{array}{l}\text { lowa, } \\
\text { USA }\end{array}$ & 12,3 & k.A. \\
\hline $\begin{array}{l}\text { Willems et al. } \\
(2005)\end{array}$ & $\begin{array}{l}\text { 24-35-Monatige } \\
(n=384)\end{array}$ & $\begin{array}{l}\text { Ghent, } \\
\text { Belgien }\end{array}$ & 18,5 & k.A. \\
\hline
\end{tabular}

k.A. = Keine Angabe 


\subsubsection{Prävalenz der frühkindlichen Karies in Deutschland}

Beim Betrachten der Datenlage zur Epidemiologie der ECC in Deutschland wird deutlich, dass nur in wenigen Studien die Erkrankungsverbreitung im Alter von 13-36 Monaten untersucht wurde (Tab. 3). Es gibt bislang keine repräsentativen, landesweiten Erhebungen in dieser Altersgruppe.

Tab. 3: Übersicht zur Prävalenz der ECC in der Bundesrepublik Deutschland

\begin{tabular}{|c|c|c|c|c|}
\hline Studie & Stichprobenauswahl & Region & $\begin{array}{c}\text { Kariesprävalenz } \\
(\%)\end{array}$ & $\begin{array}{l}\text { Kariesbefall } \\
\text { (dmft) }\end{array}$ \\
\hline $\begin{array}{l}\text { Baden und } \\
\text { Schiffner (2008) }\end{array}$ & $\begin{array}{l}\text { 3-6-Jährige, } \\
(n=1008)\end{array}$ & $\begin{array}{l}\text { Steinburg, } \\
\text { SH }\end{array}$ & 32,5 & 1,3 \\
\hline $\begin{array}{l}\text { Berndt und } \\
\text { Splieth (2009) }\end{array}$ & $\begin{array}{l}\text { 0-3-Jährige, } \\
(\mathrm{n}=395)\end{array}$ & $\begin{array}{l}\text { Greifswald, } \\
\text { MV }\end{array}$ & 12,9 & $\begin{array}{c}0,7 \\
\text { (3-Jährige) }\end{array}$ \\
\hline $\begin{array}{l}\text { Boemanns et al. } \\
\text { (1997) }\end{array}$ & $\begin{array}{l}\text { 2-3-Jährige, } \\
(n=314)\end{array}$ & $\begin{array}{l}\text { Gießen, } \\
\mathrm{HE}\end{array}$ & 13,6 & 0,5 \\
\hline Born et al. (2005) & $\begin{array}{l}\text { 3-Jährige, } \\
(\mathrm{n}=974)\end{array}$ & Hessen & 19,9 & 0,6 \\
\hline $\begin{array}{l}\text { Borutta et al. } \\
(2002)\end{array}$ & $\begin{array}{l}\text { 30-Monatige, } \\
(n=155)\end{array}$ & $\begin{array}{l}\text { Erfurt, } \\
\text { TH }\end{array}$ & 22,0 & 0,6 \\
\hline $\begin{array}{l}\text { Gräßler et al. } \\
\text { (1998) }\end{array}$ & $\begin{array}{l}\text { 18-Monatige, } \\
(n=409)\end{array}$ & $\begin{array}{l}\text { Dresden, } \\
\text { SN }\end{array}$ & 7,0 & 0,1 \\
\hline $\begin{array}{l}\text { Gülzow und } \\
\text { Farshi (2000) }\end{array}$ & $\begin{array}{l}\text { 3-6-Jährige, } \\
(n=1716)\end{array}$ & Hamburg & 34,1 & 1,5 \\
\hline $\begin{array}{l}\text { MASGF } \\
\text { Brandenburg } \\
(2008) \\
\end{array}$ & 5-Jährige & Brandenburg & 43,2 & 1,8 \\
\hline Nies et al. (2008) & $\begin{array}{l}\text { 3-7-Jährige, } \\
(n=307)\end{array}$ & Mittelhessen & 41,7 & 1,8 \\
\hline $\begin{array}{l}\text { Pieper und } \\
\text { Jablonski- } \\
\text { Momeni (2008) }\end{array}$ & $\begin{array}{l}\text { 5-7-Jährige, } \\
(n=1081)\end{array}$ & $\begin{array}{l}\text { Waldeck- } \\
\text { Frankenberg, } \\
\text { HE }\end{array}$ & 45,0 & 1,9 \\
\hline $\begin{array}{l}\text { Robke und } \\
\text { Buitkamp (2002) }\end{array}$ & $\begin{array}{l}\text { 3-4-Jährige, } \\
(n=1001)\end{array}$ & $\begin{array}{l}\text { Hannover, } \\
\mathrm{NI}\end{array}$ & 26,8 & 1,3 \\
\hline $\begin{array}{l}\text { Senkel und } \\
\text { Heinrich-Weltzien } \\
\text { (2008) }\end{array}$ & $\begin{array}{l}\text { 3-Jährige, } \\
(n=290)\end{array}$ & $\begin{array}{l}\text { Ennepe-Ruhr } \\
\text { Kreis, } \\
\text { NW }\end{array}$ & 15,5 & 0,7 \\
\hline
\end{tabular}

Das Problem bei der Prävention der frühkindlichen Karies in Deutschland ist die Polarisation auf eine kleine Bevölkerungsgruppe, die durch einen niedrigen 
Sozialstatus gekennzeichnet ist. So war im Jahr 2003 im Landkreis Steinburg 84,4\% der Karieslast auf 17,8\% der Kinder konzentriert (Baden und Schiffner 2008) und in einer Erhebung im Ennepe-Ruhr Kreis 2006 vereinten nur 12\% der Kinder 95\% des Gesamtkariesbefalls auf sich (Senkel und Heinrich-Weltzien 2008).

Weiterhin werden gravierende Mängel der zahnärztlichen Versorgung von Klein- und Vorschulkindern im gesamten Bundesgebiet beobachtet. So stellten Nies et al. (2008) bei ihrer Untersuchung in Mittelhessen fest, dass bei $71,4 \%$ der kariös betroffenen Kinder ein Behandlungsbedarf bestand. Nur 21,4\% der kariösen Zähne waren mit Füllungen versorgt und 7,2\% waren bereits extrahiert. Ein ähnliches Bild zeigte die Untersuchung von Robke und Buitkamp (2002) in Hannover. Unter den untersuchten 3-4-Jährigen betrug der Sanierungsgrad (Anteil gefüllter oder extrahierter Zähnen am gesamten dmft) lediglich 16,7\%. Auch Baden und Schiffner (2008) ermittelten im Landkreis Steinburg einen Sanierungsgrad von lediglich 19\% bei 3-Jährigen. Obwohl der Anteil sanierter Milchzähne mit zunehmendem Alter ansteigt, liegt deren Anteil deutschlandweit bei den 6-7-Jährigen immer noch nur zwischen 45,3 und 60\% (Pieper und Jablonski-Momeni 2008).

\subsection{Prävention der frühkindlichen Karies}

Um die frühkindlichen Karies zu vermeiden, werden verschiedene Ansätze einer frühzeitig einsetzenden Prävention im Schriftum empfohlen. Eine wesentliche Empfehlung ist die Reduktion der Saugerflaschengabe und die frühzeitige Gewöhnung der Kinder an eine Tassennutzung. Entscheidend ist jedoch, dass die frequente Aufnahme hoch kalorischer Getränke mit der Saugerflasche und die nächtliche Saugerflaschengabe unterbunden wird (Gussy et al. 2006, EAPD 2008, AAPD 2012). Süßigkeiten sollten, wenn überhaupt, nur zu den Hauptmahlzeiten angeboten werden.

Ein wesentlicher Aspekt der Präventionsansätze ist auch, die Eltern frühzeitig über die Kariesentstehung und mögliche Risikofaktoren zu informieren, um falsche Verhaltensweisen aus Unwissenheit zu vermeiden. Dies kann durch Gesundheitsförderung (,anticipatory guidance“) sowie Schwangeren-, Wöchnerinnen-, oder Elternberatungen durch Zahnärzte, Kinderärzte oder Hebammen geschehen (Ammari et al. 2007, Plutzer und Spencer 2008, Wagner et al. 2011). Einen besonders positiven Effekt zeigten aufsuchende Konzepte, bei denen die Gesundheitsaufklärung im häuslichen Umfeld stattfand (Twetman 2008). 
Dabei müssen die Eltern über die Übertragungswege von Mutansstreptokokken aufgeklärt werden, um eine frühe Infektion des Kindes zu vermeiden und die Mundhygiene der Eltern selbst ist zu verbessern, um das Infektionsniveau von Mutansstreptokokken im Speichel zu reduzieren (Gussy et al. 2006, EAPD 2008, AAPD 2012). Es wird weiterhin empfohlen, das Kind innerhalb der ersten zwei Lebensjahre beim Zahnarzt vorzustellen, um das Kariesrisiko einzuschätzen, das Kind an das Umfeld in der Praxis zu gewöhnen und dem Zahnarzt die Möglichkeit zu geben, weitere Präventionsmaßnahmen einzuleiten (EAPD 2008, AAPD 2012). Darüber hinaus wurde festgestellt, dass Eltern häufiger präventive Maßnahmen in Anspruch nehmen, wenn ihr Kind bereits im ersten Lebensjahr einem Zahnarzt vorgestellt wurde (Lee et al. 2006).

Eine weitere wichtige Maßnahme zur Prävention der ECC ist das tägliche Zähneputzen ab Durchbruch des ersten Zahnes mit einem Hauch und bei Kindern ab 2 Jahren mit einer erbsengroßen Menge, fluoridhaltiger Kinderzahnpasta (Twetman 2008, EAPD 2008). Dabei sollte darauf geachtet werden, dass das Kind die Zahnpasta ausspuckt und auf das Ausspülen mit Wasser nach dem Zähneputzen verzichtet wird, um eine lang anhaltende Wirkung der Fluoridionen zu gewährleisten (Oliveira et al. 2006). Kinder mit einem erhöhten Kariesrisiko sollten 2 bis 4-mal jährlich eine professionelle Fluoridlackapplikation erhalten, um einer ECCEntwicklung effektiv vorzubeugen (Weintraub et al. 2006, EAPD 2008, AAPD 2011). Eine pränatale Fluoridgabe durch die mütterliche Einnahme von Fluoridtabletten während der Schwangerschaft zeigt hingegen keinerlei kariespräventiven Effekt (Ammari et al. 2007). 


\section{Ziele der Arbeit}

Da bislang nur regional begrenzte Daten zur Kariesprävalenz und zum Kariesbefall bei 1-3-Jährigen in Deutschland vorliegen, war es das Ziel der vorliegenden Arbeit eine repräsentative Stichprobe von 1-3-Jährigen, die die Gesamtheit der Kinder dieser Altersgruppe im Bundesland Brandenburg abbilden, zu untersuchen.

Dabei sollte neben der Ermittlung der Kariesprävalenz und des Kariesbefalls bei 1-3jährigen Kindern, die eine Kita besuchen oder von einer Tagesmutter/-vater betreut werden, auch die Abhängigkeit des Kariesrisikos vom Gesundheitsverhalten und Sozialstatus der Eltern untersucht werden. Mit der Befragung der Eltern sollten Daten zu den Zahnputzgewohnheiten des Kindes, der Fluoridaufnahme, dem Ernährungsverhalten, dem häuslichen Umfeld und dem Sozialstatus der Eltern gewonnen werden. Die Verknüpfung der soziodemographischen und Mundgesundheitsdaten sollte zur Analyse von Risikofaktoren für die ECCEntstehung herangezogen werden, um für das Bundesland Brandenburg zielgerichtete Präventionsprogramme zu konzipieren, bzw. bereits etablierte Programme auf ihre Ausrichtung hin zu prüfen.

Es sollte weiterhin untersucht werden, ob die in anderen Studien genannten Risikofaktoren einer ECC auch bei 1-3-Jährigen im Bundesland Brandenburg relevant sind.

Die Fokussierung auf Kita-Kinder zielt auf die Konzeption setting-basierter Präventionsansätze, die einen direkten Einfluss auf den Alltag der Kleinkinder in der Kita nehmen. Durch die zielgerichtete Prävention Karies verursachender Verhaltensweisen insbesondere bei Kindern aus Risikogruppen, soll die Kariesprävalenz reduziert und eine sozial bedingte gesundheitliche Benachteiligung kompensiert werden.

Um diese Ziele realisieren zu können, wurde die Studie in Zusammenarbeit mit den Zahnärztlichen Diensten (ZÄD), dem Landesgesundheitsamt Brandenburg und dem Gesundheitsministerium des Landes Brandenburg durchgeführt. Zudem wurde das Projekt von der Landeszahnärztekammer Brandenburg befürwortet. 


\section{$4 \quad$ Publizierte Originalarbeit}

Frühkindliche Karies und assoziierte Risikofaktoren bei Kleinkindern im Land Brandenburg.

Deichsel M, Rojas G, Lüdecke K, Heinrich-Weltzien R.

Bundesgesundheitsblatt 55 (11-12): 1504-1511, 2012 
Bundesgesundheitsbl 2012 $\cdot 55: 1504-1511$

DOI 10.1007/s00103-012-1537-9

Online publiziert: 21. Oktober 2012

๑) Springer-Verlag 2012

\author{
M. Deichsel ${ }^{1} \cdot$ G. Rojas ${ }^{2} \cdot \mathrm{K}$. Lüdecke ${ }^{3} \cdot$ R. Heinrich-Weltzien ${ }^{1}$ \\ ${ }^{1}$ Poliklinik für Präventive Zahnheilkunde und Kinderzahnheilkunde, Universitätsklinikum Jena \\ 2 Fachbereich Jugend, Soziales und Gesundheit, Fachgruppe Gesundheit, \\ Zahnärztlicher Dienst, Stadt Brandenburg an der Havel \\ ${ }^{3}$ Abteilung Gesundheit, Referat Gesundheitsberichterstattung und Infektionsschutz, \\ Landesamt für Umwelt, Gesundheit und Verbraucherschutz Brandenburg
}

\title{
Frühkindliche Karies und assoziierte Risikofaktoren bei Kleinkindern im Land Brandenburg
}

ein Migrationshintergrund [11], ein niedriger Bildungsgrad der Mutter [12, 13, 14], ein junges Alter der Mutter [15], die frequente Verabreichung zuckerhaltiger Getränke mit der Saugerflasche, insbesondere in der Nacht $[4,14]$, eine unzureichende Zahnpflege ab dem ersten Zahn [4] und das Aufwachsen in Haushalten mit nur einem Elternteil [16]. Prolongiertes Stillen wird hinsichtlich des negativen Einflusses auf die Zahngesundheit kontrovers diskutiert [10, 17]. Als Spätfolgen der ECC wurde ein negativer Einfluss auf die kindliche Entwicklung, auf die schulische Leistungsfähigkeit und auf das soziale Verhalten aufgezeigt [18]. Weiterhin ist die Infektanfälligkeit der Kinder erhöht [19]. Die in der Regel nur unter Allgemeinanästhesie durchführbare Behandlung ist mit hohen Kosten für das Gesundheitssystem verbunden [18].

Evidenzbasierte Präventionsmaßnahmen sind das tägliche Zähneputzen mit einer fluoridhaltigen Kinderzahnpasta ab dem ersten Zahn, die 2-mal jährliche Anwendung von Fluoridlacken und eine zahngesunde Ernährung mit Verzicht auf zuckerreiche Zwischenmahlzeiten via Saugerflasche (vor allem in der Nacht; [20, 21, 22]).

Hebammenschulungen und die Aufklärung von Schwangeren über die Ätiologie und Prävention der ECC im häuslichen Umfeld sind ebenfalls Erfolg versprechende Präventionsansätze [23].

Das Ziel der vorliegenden Untersuchung war es, die Kariesprävalenz und den Kariesbefall bei 13 bis 36 Monate alten Kindern im Bundesland Brandenburg unter Berücksichtigung des Gesundheitsverhaltens und des Sozialstatus der Eltern zu untersuchen und mögliche Risikofaktoren für die Entstehung der ECC aufzudecken. Die Ergebnisse dieser Untersuchung sollen dazu beitragen, bereits bestehende und in Planung befindliche Präventionsprogramme zu bestätigen bzw. weiterzuentwickeln.

\section{Methoden}

\section{Selektion der Stichprobe}

Zehn Landkreise/kreisfreie Städte von insgesamt 18 Kreisen, die die gesamte territoriale Breite des Bundeslandes Brandenburg repräsentieren, wurden im Schuljahr 2009/2010 für das Kooperationsprojekt zwischen dem Universitätsklinikums Jena und dem Landesamt für Umwelt, Gesundheit und Verbraucherschutz des Landes Brandenburg (LUGV) zur Erfassung von Risikofaktoren der ECC selektiert. Im Schuljahr 2008/2009 wiesen der Gebisszustand und der Kariesbefall (dmft) der Kindergartenkinder sowie die Angaben der Eltern zur Erwerbstätigkeit als Indikator der sozialen Lage in diesen 10 Regionen im Vergleich zu den nicht beteiligten keine signifikanten Unterschiede auf.

Insgesamt wurden 10.161 Kinder im Alter zwischen 13 und 36 Monaten, die in einer Kindertagesstätte (Kita) oder 
Tab. 1 Vergleich von Kariesprävalenz und -befall in der Gesamtpopulation ( $n=10.161)$ und der Stichprobe mit Elternbefragung von 13 bis 36 Monate alten Kindern in 10 ausgewählten Kreisen des Landes Brandenburg

$\begin{array}{llllll}\text { Anzahl } & \text { Kariesprävalenz } & \text { Kariesbefall } & \text { Anzahl } & \text { Kariesprä- } & \text { Kariesbefall } \\ \text { Kinder } & \text { d1-4/d3-4- } & \text { d1-4mft } & \text { Kinder } & \text { valenz d1-4/ } & \text { d1-4mft } \\ \text { (n) } & \text { Niveau } & x \pm S D & \text { (N) } & \text { d3-4-Niveau } & \text { x } \pm \text { SD }\end{array}$

(\%)

(\%)

\begin{tabular}{|c|c|c|c|c|c|c|}
\hline & \multicolumn{3}{|c|}{ Gesamtpopulation (10 Kreise) } & \multicolumn{3}{|c|}{ Stichprobe mit Elternbefragung } \\
\hline & 10.161 & $5,8 / 4,1^{\text {a }}$ & $0,2 \pm 1,0^{b}$ & 661 & $7,4 / 5,3^{a}$ & $0,3 \pm 1,2^{b}$ \\
\hline Weiblich & $4912^{c}$ & $5,4 / 3,6$ & $0,2 \pm 1,0$ & $312^{c}$ & $5,4 / 3,2$ & $0,1 \pm 0,7$ \\
\hline Männlich & $5249^{c}$ & $6,2 / 4,4$ & $0,2 \pm 1,1$ & $349^{c}$ & $9,2 / 7,2$ & $0,3 \pm 1,4$ \\
\hline \multicolumn{7}{|c|}{13 bis 24 Monate alte Kinder } \\
\hline & $4085^{c}$ & $2,0 / 1,2$ & $0,1 \pm 0,6$ & $293^{c}$ & $2,4 / 1,7^{d}$ & $0,1 \pm 0,6$ \\
\hline Weiblich & 1994 & $1,9 / 1,0$ & $0,1 \pm 0,6$ & 135 & $2,2 / 1,5$ & $0,1 \pm 0,7$ \\
\hline Männlich & 2091 & $2,1 / 1,4$ & $0,1 \pm 0,6$ & 158 & $2,5 / 1,9$ & $0,1 \pm 0,5$ \\
\hline \multicolumn{7}{|c|}{25 bis 36 Monate alte Kinder } \\
\hline & $6076^{c}$ & $8,4 / 6,0$ & $0,3 \pm 1,2$ & $368^{c}$ & $11,4 / 8,2^{d}$ & $0,4 \pm 1,4$ \\
\hline Weiblich & 2918 & $7,7 / 5,5$ & $0,3 \pm 1,1$ & 177 & $7,9 / 4,5$ & $0,2 \pm 0,7$ \\
\hline Männlich & 3158 & $9,0 / 6,4$ & $0,3 \pm 1,3$ & 191 & $14,7 / 11,5$ & $0,6 \pm 1,8$ \\
\hline
\end{tabular}

Kein signifikanter Unterschied zwischen Gesamtpopulation und Stichprobe: In der Kariesprävalenz, $p=0,116$. bIn dmft-Werten, $p=0,111$. In der Alters- und Geschlechterverteilung, $p=0,094$. ${ }^{d}$ Signifikanter Unterschied in der Kariesprävalenz zwischen 13 bis 24 und 25 bis 36 Monate alten Kindern, $p=0,001$.

Tab. 2 Kariesbefall auf Dentinkaries-(d3-4)-Niveau sowie Anzahl kariöser Zähne mit Schmelzkaries (d1-2t) und Dentinkaries (d3-4t) von 13 bis 36 Monate alten Kindern in 10 ausgewählten Kreisen des Landes Brandenburg

\begin{tabular}{|c|c|c|c|c|c|c|c|}
\hline & $\begin{array}{l}\text { Anzahl } \\
\text { Kinder } \\
\text { (n) }\end{array}$ & $\begin{array}{l}\text { Anzahl } \\
\text { Zähne } \\
x \pm S D\end{array}$ & $\begin{array}{l}\text { Anzahl } \\
\text { gesunder } \\
\text { Zähne } \\
\text { x } \pm \text { SD }\end{array}$ & $\begin{array}{l}\text { d3-4mft } \\
x \pm S D\end{array}$ & $\begin{array}{l}d 1-2 t \\
x \pm S D\end{array}$ & $\begin{array}{l}d 3-4 t \\
x \pm S D\end{array}$ & $\begin{array}{l}\mathrm{SiC} \text { (d1- } \\
4 \mathrm{mft}) \\
\mathrm{x} \pm \mathrm{SD}\end{array}$ \\
\hline & 661 & $16,5 \pm 3,9$ & $16,3 \pm 3,9$ & $0,2 \pm 1,0$ & $0,1 \pm 0,4$ & $0,2 \pm 0,9$ & $0,8 \pm 2,0$ \\
\hline Weiblich & 312 & $16,5 \pm 3,9$ & $16,4 \pm 3,9$ & $0,1 \pm 0,6$ & $0,1 \pm 0,3$ & $0,1 \pm 0,5$ & $0,5 \pm 1,2$ \\
\hline Männlich & 349 & $16,6 \pm 3,8$ & $16,2 \pm 3,9$ & $0,3 \pm 1,2$ & $0,1 \pm 0,4$ & $0,2 \pm 1,1$ & $1,1 \pm 2,4$ \\
\hline \multicolumn{8}{|c|}{13 bis 24 Monate alte Kinder } \\
\hline & 293 & $13,8 \pm 3,9$ & $13,7 \pm 3,9$ & $0,1 \pm 0,5$ & $0,02 \pm 0,2$ & $0,1 \pm 0,5$ & $0,3 \pm 1,1$ \\
\hline Weiblich & 135 & $13,6 \pm 4,0$ & $13,5 \pm 4,0$ & $0,1 \pm 0,6$ & $0,02 \pm 0,2$ & $0,1 \pm 0,6$ & $0,3 \pm 1,2$ \\
\hline Männlich & 158 & $13,9 \pm 3,8$ & $13,9 \pm 3,8$ & $0,1 \pm 0,5$ & $0,01 \pm 0,1$ & $0,1 \pm 0,5$ & $0,2 \pm 0,9$ \\
\hline \multicolumn{8}{|c|}{25 bis 36 Monate alte Kinder } \\
\hline & 368 & $18,7 \pm 2,0$ & $18,4 \pm 2,3$ & $0,3 \pm 1,2$ & $0,1 \pm 0,5$ & $0,2 \pm 1,1$ & $1,3 \pm 2,4$ \\
\hline Weiblich & 177 & $18,7 \pm 1,8$ & $18,5 \pm 1,9$ & $0,1 \pm 0,6$ & $0,1 \pm 0,4$ & $0,1 \pm 0,5$ & $0,6 \pm 1,2$ \\
\hline Männlich & 191 & $18,7 \pm 2,1$ & $18,2 \pm 2,6$ & $0,5 \pm 1,6$ & $0,1 \pm 0,5$ & $0,4 \pm 1,4$ & $1,9 \pm 3,0$ \\
\hline
\end{tabular}

Tagespflegestätte betreut wurden, untersucht. In einer Stichprobe von 94 Kitas, die von den Zahnärztlichen Diensten (ZÄD) der Gesundheitsämter unter Berücksichtigung der landesspezifischen sozioökonomischen Situation selektiert wurden, erfolgte neben der Erfassung der Mundgesundheit eine Elternbefragung zum Gesundheitsverhalten und Sozialstatus. Die ZÄD übernahmen aus Datenschutzgründen die Personalisierung der Elternfragebögen der Kinder dieser Altersgruppen; insgesamt wurden 1128 Fragebögen ausgehändigt; 675

\section{Elternfragebogen}

Die 22 Fragen des Elternfragebogens orientierten sich an den von der AAPD genannten Risikofaktoren und Präventionsempfehlungen zur ECC [24, 25]. Die Fragen zu den Mundhygienegewohnheiten umfassten Angaben zum Zähneputzen wie Putzbeginn, Häufigkeit des Putzens, Putzen mit eigener Zahnbürste und Unterstützung bzw. Nachputzen der Eltern sowie Fragen zu den Ernährungsgewohnheiten des Kindes, die vorrangig auf die Verabreichung der Saugerflasche, insbesondere die nächtliche Flaschengabe, abzielten. Weiterhin wurde die Verwendung von fluoridhaltiger Kinderoder Erwachsenenzahnpasta, fluoridiertem Speisesalz und Fluoridtabletten erfragt. Weitere Fragen zielten auf die Ermittlung des Sozialstatus der Eltern ab, wobei die Erfassung des Schulabschlusses und der Erwerbstätigkeit der Eltern eine Einstufung nach dem Brandenburger Sozialindex erlaubte [26]. Der konzipierte Fragebogen wurde vor Untersuchungsbeginn mit jungen Müttern auf seine Verständlichkeit geprüft; nach ihren Empfehlungen wurden Änderungen vorgenommen.

Das Anschreiben an die Eltern, das die schriftliche Einwilligung der Erziehungsberechtigten zur anonymisierten Auswertung der Daten einschloss, wurde in Abstimmung mit dem Ministerium für Umwelt, Gesundheit und Verbraucherschutz des Landes Brandenburg erstellt, das die Befragung genehmigte.

\section{Zahnärztliche Untersuchung}

Die zahnärztliche Untersuchung der Kinder wurde von kalibrierten Zahnärzten der ZÄD im Rahmen der gruppenprophylaktischen Betreuung gemäß $₫ 21$ SGB V im Zeitraum von September 2009 bis Juli 2010 in den Kitas oder Tagespflegestätten durchgeführt. Der Kariesbefall wurde auf Schmelz-(d1-4)- und Dentinkaries-(d3-4)-Niveau nach den kariesdiagnostischen Kriterien der WHO [27] und dem „Leitfaden für Zahnärztliche Dienste der Gesundheitsämter im Land Brandenburg" erfasst [28]. Als Schmelzkaries ist eine initiale, jedoch remineralisierbare Karies definiert; eine Dentin- 
karies ist eine ausschließlich restaurativ zu behandelnde kavitierte Karies [27, 28]. Die Kinder wurden nach dem Zähneputzen stehend oder auf dem Schoß der Erzieher/-innen mit Mundspiegel und Sonde befundet.

Die Untersuchung wurde im „Zahnärztlichen Prophylaxe-Pass Vorschulkinder", den jedes Kind ab dem 2. Lebensjahr erhält, vermerkt. Die kariesepidemiologischen Befunde wurden im Modul „Zahnärztlicher Dienst“ einer Standardsoftware der Gesundheitsbehörden dokumentiert.

\section{Statistische Auswertung}

Der Kariesbefall wurde zahnbezogen als d1-4mft-Index berechnet [29]. Der Significant Caries Index (SiC) wurde herangezogen, um die Polarisation des $\mathrm{Ka}$ riesbefalls in der Population zu beschreiben [30]. Die Schwere des Kariesbefalls wurde mit der ECC-Klassifikation nach Wyne kategorisiert [31]. Weiterhin wurden der Sanierungsgrad und der Füllungsindex berechnet.

Die Auswertung der Daten erfolgte mit der Statistiksoftware SPSS 19. Die Repräsentativität der Stichprobe mit der Fragebogenerhebung zur Gesamtstichprobe der untersuchten Kinder in den 10 Kreisen wurde mit dem $\chi^{2}$-Test geprüft. Der Zusammenhang zwischen dem Kariesbefall und den Fragebogenvariablen wurde für jede Frage zuerst bivariat geprüft. Dazu wurde für jede potenzielle Einflussgröße getrennt ein binäres logistisches Modell gerechnet. Die potenziellen Einflüsse wurden indikatorkodiert in das Modell gegeben. Variablen, die bei Verwendung des $\chi^{2}$-Tests (Wald-Statistik) einen signifikanten $\mathrm{Zu}$ sammenhang zum Kariesbefall aufwiesen, wurden mit einer schrittweisen Variablenauswahl in die multiple binäre logistische Regression eingeschlossen. Vorher wurde das Modell alters- und geschlechtsadjustiert. Merkmale, die nach der bivariaten Analyse ausgeschlossen waren, wurden in einem zweiten Block noch einmal dem Modell angeboten, erreichten aber auch hier keine Signifikanz. Das Signifikanzniveau wurde mit einer Irrtumswahrscheinlichkeit von $\mathrm{p} \leq 0,05$ festgelegt.

Bundesgesundheitsbl 2012 ·55:1504-1511 DOI 10.1007/s00103-012-1537-9

c) Springer-Verlag 2012

\section{Deichsel · G. Rojas · K. Lüdecke · R. Heinrich-Weltzien \\ Frühkindliche Karies und assoziierte Risikofaktoren bei Kleinkindern im Land Brandenburg}

\section{Zusammenfassung}

Ziel der vorliegenden Studie war die Erfassung des Kariesbefalls in einer repräsentativen Stichprobe von 13 bis 36 Monate alten Kindern im Land Brandenburg in Beziehung zum Gesundheitsverhalten und Sozialstatus der Eltern; 661 Kinder aus 10 Kreisen wurden nach WHO-Standard (1997) durch kalibrierte Zahnärzte des Öffentlichen Gesundheitsdienstes untersucht. Gesundheitsverhalten und Sozialstatus der Eltern wurden fragebogenbasiert erfasst. Die Kariesprävalenz betrug 7,4\% auf Schmelzkaries(d1-4)- und 5,3\% auf Dentinkaries-(d3-4)-Niveau. Der Kariesbefall belief sich auf 0,3 d1$4 \mathrm{mft} / 0,2 \mathrm{~d} 3-4 \mathrm{mft}$. Zwei Prozent der Kinder vereinten $52 \%$ des Kariesbefalls auf sich. Nur $19,9 \%$ der Zähne waren saniert und 10\% mit einer Füllung versorgt. Fünfundzwanzig bis 36 Monate alte Kinder, Kinder mit niedrigem
Sozialstatus oder nächtlicher Flaschengabe hatten ein signifikant höheres Kariesrisiko $(\mathrm{OR} 3,84, \mathrm{p}=0,005 ; \mathrm{OR} 7,34, \mathrm{p}<0,0001$; OR $2,4, p=0,022)$ als jüngere Kinder, Kinder mit hohem Sozialstatus und ohne Flaschengabe. Zur Kompensation der sozial bedingten gesundheitlichen Benachteiligung sind frühzeitige intersektorale Präventionsprogramme in Kindertagesstätten zu favorisieren. Der niedrige Sanierungsgrad muss mit den zahnärztlichen Körperschaften kommuniziert werden, um die Zugängigkeit von Kleinkindern in die Zahnarztpraxis zu verbessern.

\section{Schlüsselwörter}

Frühkindliche Karies · Kleinkinder . Gesundheitsverhalten · Sozialstatus . Präventionsprogramme

\section{Early childhood caries and associated risk factors among infants in the German federal state of Brandenburg}

\section{Abstract}

The objective of this study was to determine the caries experience in a representative sample of infants aged 13-36 months in the federal state of Brandenburg in relation to their parents' health behavior and social status. Calibrated public health service dentists examined 661 children in ten districts according to WHO standards (1997). Data on the parents' health behavior and their social status were acquired via questionnaire. The prevalence of caries was $7.4 \%$ on enamellevel (d1-4) and 5.3\% on dentin-level (d3-4). The caries experience was $0.3 \mathrm{~d} 1-4 \mathrm{mft} / 0.2$ d3-4mft. Fifty-two percent of the total caries burden was concentrated on $2 \%$ of the children. The restorative index was $19.9 \%$ and the care index $10 \%$, respectively. Children of 25-36 months of age and with a lower so- cial status and those who were bottle-fed at night revealed a significantly higher caries risk (OR 3.84, $p=0.005$; OR 7.34, $p<0.0001$; OR 2.4, $p=0.022$ ) compared to younger children, children with a higher social status, or those without nocturnal bottle use. Intersectoral prevention programs in day-care centers are the favored preventive approach to compensate the socially determined health inequality. The low care index needs to be communicated with the dental association to improve the accessibility of infants in dental practice.

\section{Keywords}

Early childhood caries · Infants · Health behavior $\cdot$ Social status . Prevention programs
Nicht alle Merkmale, die bivariat signifikant waren, wurden in das Modell aufgenommen. Zum einen lag dies an der impliziten Kindesaltersabhängigkeit der Antworten, (die durch die Altersadjustierung korrigiert wurden), und zum anderen am starken Einfluss des elterlichen Sozialstatus auf das Gesundheitsverhalten, was zu einer Multikollineari- tät zwischen den potenziellen Prädiktoren führt. Deshalb sind neben den Ergebnissen aus der Modellrechnung auch die bivariaten Zusammenhänge von Interesse. 


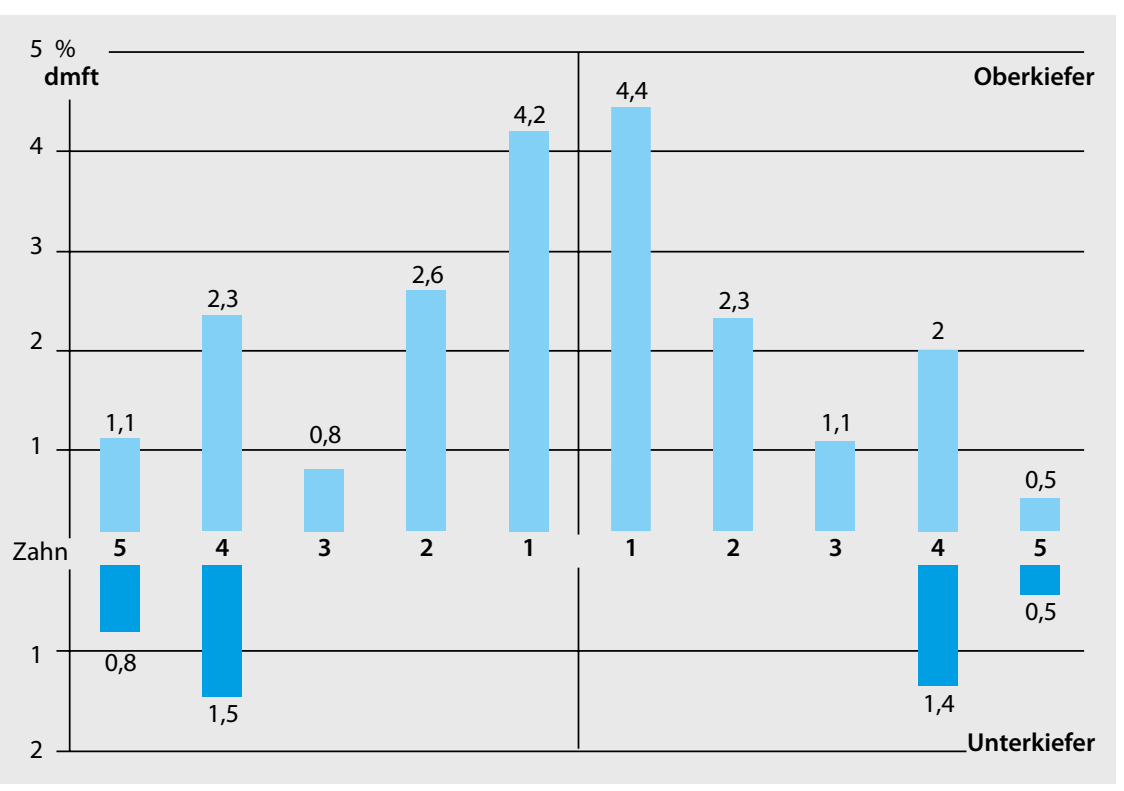

Abb. 1 Z Zahnbezogene Topografie des Kariesbefalls (Angaben in \% d1-4mft) von Kindern im Alter von 13 bis 36 Monaten in 10 ausgewählten Kreisen des Landes Brandenburg

\section{Ergebnisse}

\section{Kariesepidemiologische Untersuchung}

Die Gesamtpopulation der 13 bis 36 Monate alten Kinder in den selektierten 10 Kreisen $(\mathrm{n}=10.161)$ wies eine Kariesprävalenz von $5,8 \%$ auf Schmelzkaries(d1-4)-Niveau und von 4,1\% auf Dentinkaries-(d3-4)-Niveau auf. Der Kariesbefall betrug 0,2 ( $\pm 1,0) \mathrm{d} 1-4 \mathrm{mft}$ (• Tab. 1). In der Stichprobe mit Elternbefragung $(\mathrm{n}=661)$ lag eine Kariesprävalenz von $7,4 \%$ auf Schmelzkaries-(d1-4)-Niveau und von 5,3\% auf Dentinkaries-(d3-4)Niveau vor; 25 bis 36 Monate alte Kinder wiesen eine signifikant höhere Prävalenz auf als 13 bis 24 Monate alte Kinder. Der Kariesbefall belief sich auf $0,3( \pm 1,2)$ d1$4 \mathrm{mft}$. Da keine signifikanten Unterschiede in der Stichprobe mit Elternbefragung hinsichtlich der Alters- und Geschlechtsverteilung, Kariesprävalenz und Verteilung der dmft-Werte zur Gesamtpopulation vorlagen, ist die Repräsentativität der Stichprobe gegeben.

Der Kariesbefall auf Dentinkaries-(d3-4)-Niveau entspricht nahezu dem auf Schmelzkaries-(d1-4)-Niveau (- Tab. 2); anhand der d3-4t-Einzelkomponente des d3-4mft-Indexes wird deutlich, dass nahezu der gesamte Ka- riesbefall bei den 13 bis 24 Monate alten Kindern auf den d3-4t-Wert entfällt. Bei den 25 bis 36 Monate alten Kindern wurde ein Anstieg der Schmelzkaries (d1-2tWert) deutlich. Der SiC-Index charakterisierte mit einem mehr als doppelt so hohen Kariesbefall von $0,8( \pm 2,0)$ d1$4 \mathrm{mft}$ als dem aller 13 bis 36 Monate alten Kinder das Drittel der Population mit dem höchsten Kariesbefall; $52 \%$ des Gesamtkariesbefalls war auf $2 \%$ aller Kinder konzentriert. Die oberen Frontzähne und die ersten Milchmolaren im Oberund Unterkiefer waren am häufigsten kariös befallen (• Abb. 1). Der ECC-Typ 1, charakterisiert durch kariöse Milchmolaren, lag bei 2,6\% der Kinder vor. Der ECC-Typ 2, charakterisiert durch den Kariesbefall der oberen Frontzähne, wurde bei 4,8\% der Kinder beobachtet. 0,6\% der Kinder waren saniert, 4,7\% waren behandlungsbedürftig, und 2,1\% wiesen einen Präventionsbedarf auf. Nur 19,9\% (Sanierungsgrad) aller kariösen Zähne waren saniert, und 10\% (Füllungsindex) waren mit einer Füllung versorgt.

\section{Ergebnisse der Elternbefragung}

Die Auswertung des Elternfragebogens zeigte, dass über 99\% der Kinder eine eigene Zahnbürste besitzen und 80\% 2-mal täglich oder öfter ihre Zähne put- zen. Nur 56\% der Eltern putzten ab dem Durchbruch des ersten Milchzahnes die Zähne des Kindes; 65\% der Kinder putzten sich allein die Zähne, 89\% der Eltern putzen mindestens 1-mal täglich die Zähne ihrer Kinder nach. Eine fluoridhaltige Kinderzahnpasta verwenden 89\% der Eltern, $81 \%$ der Kinder haben in den ersten 3 Lebensjahren Fluoridtabletten erhalten, und 55\% der Haushalte verwenden fluoridiertes Speisesalz.

Lediglich $40 \%$ der Eltern waren mit ihrem Kind bereits beim Zahnarzt; von diesen hatte jedes zehnte Kind Schmerzen oder eine Überweisung vom ZÄD erhalten. 53\% der Eltern mit Zahnarztkontakt wurden über die Vorbeugung der ECC, die Verwendung von Fluoriden, den Saugerflaschengebrauch oder zahngesunde Ernährung beraten. 8\% der Kinder bekamen regelmäßig zuckerhaltige Medikamente verabreicht.

Für die Analyse der Mundgesundheitsdaten in Bezug zu den Fragebogenvariablen wurde die Population in Kinder mit und ohne Kariesbefall dichotomisiert und bivariate logistische Regressionen ohne Alters- und Geschlechtskorrektur berechnet (• Tab. 3). Dabei deuten sich erste Ergebnisse an: Bei Kindern mit nächtlicher Saugerflaschengabe ist das Kariesrisiko signifikant erhöht. Je häufiger Kinder zuckerreiche Getränke zwischen den Mahlzeiten bekommen und je jünger die Eltern sind, desto höher ist ihr Kariesrisiko. Wachsen Kinder bei einem alleinerziehenden Elternteil auf, erhöht sich ebenfalls das Kariesrisiko. Der Brandenburger Sozialindex bündelt mehrere Risikofaktoren. Kinder aus Haushalten mit einem niedrigen Sozialindex haben ein vielfach höheres Kariesrisiko als Kindern mit hohem Sozialstatus. Der erste Zahnarztbesuch kariös betroffener Kinder fand häufiger erst nach dem 2. Lebensjahr statt. Kinder, deren Eltern keine Mundgesundheitsberatung bei ihrem Zahnarzt erhielten, wiesen signifikant häufiger eine Karies auf $(p=0,007)$ als Kinder, deren Eltern beraten wurden. In der multivariaten Analyse musste auf die Fragebogenvariable zum Zahnarztbesuch jedoch verzichtet werden, da nach dem Ausschluss von Kindern mit einer positiven Schmerzanamnese und denen, die eine Aufforderung 
Tab. 3 Bivariate Regressionsanalyse des Antwortspektrums der Eltern in Bezug zum Kariesbefall ( $\mathrm{dmft}$ ) der Kinder im Alter von 13 bis 36 Monaten in 10 ausgewählten Kreisen des Landes Brandenburg

\begin{tabular}{|c|c|c|c|c|}
\hline Variable & Referenzwert & Odds Ratio (OR) & 95\%-KI & p-Wert \\
\hline \multicolumn{4}{|c|}{ Seit wann putzt Ihr Kind die Zähne? } & 0,195 \\
\hline Seit 1. Lebensjahr & Seit 1.Zahn & 1,15 & $0,61-2,20$ & 0,663 \\
\hline Seit 2. Lebensjahr & Seit 1.Zahn & 2,57 & $1,09-6,06$ & 0,031 \\
\hline Noch gar nicht & Seit 1.Zahn & 0,00 & - & 0,999 \\
\hline \multicolumn{4}{|c|}{ Wann putzt sich Ihr Kind die Zähne? } & 0,402 \\
\hline Morgens & $\begin{array}{l}\text { Morgens und } \\
\text { abends }\end{array}$ & 0,00 & - & 0,998 \\
\hline Abends & $\begin{array}{l}\text { Morgens und } \\
\text { abends }\end{array}$ & 1,09 & $0,48-2,44$ & 0,839 \\
\hline Öfter & $\begin{array}{l}\text { Morgens und } \\
\text { abends }\end{array}$ & 1,93 & $0,91-4,12$ & 0,088 \\
\hline \multicolumn{4}{|c|}{ Wie lange wurde Ihr Kind gestillt? } & 0,579 \\
\hline 0 bis 12 Monate & Gar nicht & 1,36 & $0,60-3,13$ & 0,464 \\
\hline 13 bis 24 Monate & Gar nicht & 0,00 & - & 0,998 \\
\hline 25 bis 36 Monate & Gar nicht & 5,24 & $0,48-57,09$ & 0,174 \\
\hline \multicolumn{4}{|c|}{ Wie lange bekam Ihr Kind die Flasche? } & 0,033 \\
\hline Gar nicht & 0 bis 12 Monate & 1,95 & $0,57-6,67$ & 0,288 \\
\hline 13 bis 24 Monate & 0 bis 12 Monate & 1,03 & $0,27-3,92$ & 0,964 \\
\hline 25 bis 36 Monate & 0 bis 12 Monate & 3,53 & $0,97-12,91$ & 0,057 \\
\hline \multicolumn{4}{|c|}{ Bekommt Ihr Kind nachts die Flasche? } & 0,040 \\
\hline $\mathrm{Ja}$ & Nein & 2,06 & $1,03-4,12$ & 0,040 \\
\hline \multicolumn{4}{|c|}{ Wie oft bekommt Ihr Kind zuckerreiche Getränke zwischen den Mahlzeiten? } & 0,149 \\
\hline Bis zu 3-mal pro Woche & Selten & 1,07 & $0,31-3,72$ & 0,919 \\
\hline 1-mal täglich & Selten & 1,03 & $0,40-2,63$ & 0,947 \\
\hline 2-bis 3-mal täglich & Selten & 2,02 & $0,94-4,35$ & 0,072 \\
\hline Öfter & Selten & 2,56 & $1,08-6,09$ & 0,034 \\
\hline \multicolumn{4}{|l|}{ Wie alt sind Sie? } & 0,033 \\
\hline Bis 20 Jahre & Bis 35 Jahre & 6,00 & $1,44-25,08$ & 0,014 \\
\hline Bis 25 Jahre & Bis 35 Jahre & 3,00 & $1,18-7,65$ & 0,021 \\
\hline Bis 30 Jahre & Bis 35 Jahre & 2,54 & $1,13-5,71$ & 0,024 \\
\hline Über 35 Jahre & Bis 35 Jahre & 1,30 & $0,45-3,74$ & 0,630 \\
\hline \multicolumn{4}{|c|}{ Wie viele Kinder leben in Ihrem Haushalt? } & 0,251 \\
\hline 1 & 2 & 1,30 & $0,68-2,47$ & 0,430 \\
\hline 3 & 2 & 2,32 & $0,80-6,73$ & 0,123 \\
\hline 4 und mehr & 2 & 3,50 & $0,70-17,57$ & 0,128 \\
\hline \multicolumn{4}{|c|}{ Wie viele Erwachsene leben in Ihrem Haushalt? } & 0,092 \\
\hline 1 & 2 & 1,84 & $0,91-3,75$ & 0,092 \\
\hline \multicolumn{4}{|c|}{ Brandenburger Sozialindex } & $<0,001$ \\
\hline Niedriger Sozialstatus & Hoher Sozialstatus & 8,06 & $3,37-19,26$ & $<0,001$ \\
\hline Mittlerer Sozialstatus & Hoher Sozialstatus & 2,06 & $1,01-4,21$ & 0,046 \\
\hline
\end{tabular}

zum Zahnarztbesuch von den ZÄD erhalten hatten, keine Modellrechnung mit allen kariös betroffenen Kindern möglich war.

In der multiplen logistischen Regressionsanalyse wurden aufgrund der geringen Kinderzahl mit Kariesbefall $(\mathrm{n}=49)$ die Variable "Flaschengabe“ nach "Ja“ und "Nein“ differenziert und das Alter der Eltern in den Kategorien „bis 20 Jahre“, „20 bis 30 Jahre“ und „über 30 Jahre" zusammengefasst. Die Regressionsanalyse zeigte, dass der Sozialstatus der Eltern, die nächtliche Flaschengabe und das Alter der Kinder die entscheidenden Faktoren für ein erhöhtes Kariesri- siko darstellen (• Tab.4). Das ECC-Risiko eines Kindes mit niedrigem Sozialstatus war um das 7,34-Fache höher als das eines Kindes mit hohem Sozialstatus. 25 bis 36 Monate alte Kinder und Kinder mit nächtlicher Gabe der Saugerflasche hatten ein 3,84- bzw. 2,4-fach höheres Risiko (OR), an Karies zu erkranken, als Jüngere und Kinder ohne nächtliche Flaschengabe.

\section{Diskussion}

Die vorliegende Studie ermittelte erstmalig landesweite Daten zur ECC-Prävalenz in ihrem Beziehungsgefüge zum Mundgesundheitsverhalten und Sozialstatus der Eltern. Die Kariesprävalenz und der Kariesbefall in der Stichprobe der 13 bis 36 Monate alten Kinder unterschieden sich 2010 nicht wesentlich von dem Erkrankungsaufkommen im gesamten Bundesland Brandenburg. Im Vergleich zu internationalen Studien, die eine ECC-Prävalenz bei gleichaltrigen Kindern zwischen 7 und 32\% beobachteten, liegt die Prävalenzrate im Land Brandenburg im unteren Bereich [4, 5, 6, 7]. Mit dem Lorenz-Konzentrations$\mathrm{maß}$ und dem SiC-Index wurde jedoch erstmalig die starke Polarisation des Kariesbefalls in dieser Altersgruppe aufgezeigt; $52 \%$ des Gesamtkariesbefalls war auf lediglich 2\% der Kinder konzentriert. Der Kariesbefall von dem Drittel aller Kinder mit dem höchsten Kariesbefall, war 2,7-fach höher als der der Gesamtpopulation. Sowohl der niedrige Sanierungsgrad $(19,9 \%)$ als auch Füllungsindex (10\%) bestätigen die zahnärztliche Unterversorgung von Klein- und Vorschulkindern in Deutschland [32, 33, 34]. Neben der Herausforderung der zahnärztlichen Behandlung selbst, dürfte der zu späte erste Zahnarztbesuch Ursache für diese unbefriedigende Situation sein. Je jünger die Kinder sind, umso häufiger werden sie nicht behandelt.

Diese Aussagen gelten für $62 \%$ der Kinder in einer Tagesbetreuung dieser Altersgruppe. Bei Hauskindern kann eine höhere Kariesprävalenz angenommen werden, da bei ihnen der Anteil von Familien mit niedrigem Sozialstatus deutlich höher ist, wie dies die internen Daten der Kinder- und Jugendgesund- 
Tab. 4 Multivariate Regressionsanalyse (alters- und geschlechtsadjustiert) zur Assoziation der ECC mit Verhaltens- und sozialen Variablen bei Kindern im Alter von 13 bis 36 Monaten in 10 ausgewählten Kreisen des Landes Brandenburg

\begin{tabular}{|lllll}
\hline Variable & Referenzwert & Odds Ratio (OR) & 95\%-KI & p-Wert \\
\hline Alter: 25 bis 36 Monate & 13 bis 24 Monate & $\mathbf{3 , 8 4}$ & $1,49-9,91$ & $\mathbf{0 , 0 0 5}$ \\
\hline $\begin{array}{l}\text { Jungen im Alter von 25 bis } \\
\text { 36 Monate }\end{array}$ & $\begin{array}{l}\text { Mädchen oder 13 bis } \\
\text { 24 Monate alt }\end{array}$ & 1,84 & $0,91-3,74$ & 0,090 \\
\hline Niedriger Sozialstatus & Hoher Sozialstatus & $\mathbf{7 , 3 4}$ & $\mathbf{2 , 9 6 - 1 8 , 2 1}$ & $<0,0001$ \\
\hline Mittlerer Sozialstatus & Hoher Sozialstatus & 1,94 & $0,94-4,02$ & 0,075 \\
\hline Nächtliche Flaschengabe & Keine & $\mathbf{2 , 4 0}$ & $1,13-5,08$ & $\mathbf{0 , 0 2 2}$ \\
\hline $\begin{array}{l}\text { Signifikante Variablen sind fett hervorgehoben. } \\
\text { KI Konfidenzintervall. }\end{array}$ & & & \\
\hline
\end{tabular}

heitsdienste der Pflichtuntersuchung von 30 bis 42 Monate alten Kindern zeigen. Es sind 39\% der Eltern von Hauskindern nicht erwerbstätig, bei Eltern von Kita-Kindern liegt dieser Anteil bei 9\%.

Ein niedriger Sozialstatus und die nächtliche Gabe der Saugerflasche wurden als Hauptrisikofaktoren für eine ECC bei den brandenburgischen Kindern identifiziert. Die signifikante Assoziation der ECC mit der Dauer und der nächtlichen Flaschengabe, einem jungen Alter der Eltern und dem kindlichen Aufwachsen bei alleinerziehenden Elternteilen in der bivariaten Analyse waren in der multivariaten Regressionsanalyse nicht signifikant. Daher sind multivariate statistische Verfahren für die Aufdeckung wesentlicher Assoziationen aufgrund der multifaktoriellen ECC-Ätiologie unumgänglich. Die gleichen Assoziationen wurden international ebenfalls aufgezeigt $[2,4,10,12,13$, $14,15,16,17,22,35]$. Hallet und Rourke [15] fanden ein 1,9-fach und Mohebbi et al. [17] ein 5,5-fach erhöhtes ECC-Risiko (OR) bei nächtlicher Flaschengabe, während in unserer Stichprobe ein OR von 2,4 ermittelt wurde. Nicht überraschend war hingegen, dass 25 bis 36 Monate alte Kinder ein höheres Kariesrisiko aufwiesen als jüngere, da bekanntermaßen der Kariesbefall mit zunehmendem Alter ansteigt, wenn kein gesundheitsförderndes Verhalten praktiziert wird. Dass ein niedriges Bildungsniveau der Eltern einen Risikofaktor für die ECC darstellt, wurde in jüngeren Untersuchungen mit einer $\mathrm{OR}$ von 2,2 [4], 2,37 [22] und 1,64 [13] belegt. In der vorliegenden Untersuchung ist die Bildung der Eltern im Brandenburger Sozialindex eingeschlossen [26]. Ein niedriger Sozialstatus der Kinder war im Vergleich zu einem hohen mit einer 7,34-fachen OR, an einer ECC zu erkranken, assoziiert.

Eine ECC-Prävention ist ohne Fluoride nicht möglich. Dabei weisen die lokale Fluoridapplikation und Verwendung fluoridhaltiger Zahnpasta im Gegensatz zur pränatalen Gabe von Fluoridtabletten einen kariesprotektiven Effekt auf [24]. Zähneputzen ab dem ersten Zahn mit einer 500 ppm fluoridhaltigen Zahnpasta bis zum 2. Lebensjahr und danach mit mindestens einer 1000 ppm Fluoridzahnpasta wird als evidenzbasierte Maßnahme von der European Academy of Peadiatric Dentistry (EAPD) empfohlen [21]. In der deutschen Leitlinie „Fluoridierungsmaßnahmen" wird als Basisprävention die tägliche Verwendung einer Fluoridzahnpasta und die häusliche Nutzung von fluoridhaltigem Speisesalz empfohlen [38]. In unserer Befragung gab nur etwa die Hälfte (55\%) aller Eltern an, fluoridhaltiges Speisesalz zu verwenden, während $89 \%$ aller Befragten eine Fluoridzahnpasta zur Zahnpflege ihrer Kinder nutzen. Bei Kindern mit einem erhöhten Kariesrisiko ist die 2-mal jährliche Applikation von Fluoridlacken als ergänzende Präventionsmaßnahme indiziert $[20,25,37]$. In der vorliegenden Studie bestand bei 2,1\% der Kinder ein solcher Präventionsbedarf. Dem Kariesbefallsmuster zufolge sind die ersten Milchmolaren und oberen Frontzähne prioritär zu behandeln. Für die lokale Fluoridapplikation hoch konzentrierter Präparate wurde eine Kariesreduktion von etwa $10 \%$ nachgewiesen [38].

Neben der Verfügbarkeit von Fluoriden tragen die Gesundheitsfrühförderung und Aufklärung von Schwangeren und jungen Müttern wesentlich zur
ECC-Prävention bei $[23,39,40]$. Die frühzeitige präventive Intervention hat jedoch nur dann Erfolg, wenn sie durch eine kontinuierliche und risikoorientierte zahnärztliche Betreuung flankiert wird. In unserer Stichprobe waren lediglich $40 \%$ der Kinder beim Zahnarzt. Etwa die Hälfte der Eltern (47\%) hatte keine zahnärztliche Beratung zur ECC-Prävention erhalten und wiesen signifikant häufiger eine Karies auf $(p=0,007)$. Die Aufklärung und Beratung von Schwangeren sowie die konsequente Umsetzung der in den zahnärztlichen Frühuntersuchungen (FU) vorgesehenen Ernährungsund Mundhygieneberatungen der Erziehungsberechtigten ist aus Sicht der vorliegenden Evidenz auch eine ethische Pflicht des zahnärztlichen Berufsstandes [41]. Darüber hinaus ist die Einführung der FU vor dem 30. Lebensmonat notwendig, wenn dem frühen ECC-Befall Rechnung getragen werden soll [35]. Diese dürfte neben den positiven Auswirkungen auf die Mundgesundheit der Kinder auch erhebliche Kosteneinsparungen mit sich bringen, da Eltern häufiger präventive Leistungen in Anspruch nehmen, wenn ihre Kinder bereits im 1. Lebensjahr dem Zahnarzt vorgestellt werden [42]. Weiterhin ist es unumgänglich, die Pädiater in ein intersektorales Präventionsprogramm einzubeziehen, da sie bislang die einzigen sind, die die Kinder in den ersten Lebensjahren im Rahmen der U-Untersuchungen betreuen. Aus zahnärztlicher Sicht muss darüber hinaus die gruppenprophylaktische Betreuung der Kleinkinder nach $\$ 21$ SGB V in den Kitas umgesetzt werden. Im Bundesland Brandenburg wurden hierzu Konzepte entwickelt, die als begleitende Gesundheitsförderung die Einbeziehung der Eltern in die ECCPrävention ab dem 1. Milchzahn in Kitas und Tagespflegestätten zum Ziel haben. Der „Zahnärztliche Prophylaxe-Pass Mutter \& Kind" klärt über Risiken der Mundgesundheit in der Schwangerschaft und im Kleinkindalter auf und wird den Schwangeren mit dem Mutterpass von den Gynäkologen ausgehändigt. Schulungen für Familienpaten in den Netzwerken "Gesunde Kinder" ergänzen diesen intersektoralen Präventionsansatz. $\mathrm{Ab}$ dem 2. Lebensjahr erhalten alle Kin- 
der in Kitas und Tagespflegestätten den „Zahnärztlichen Prophylaxe-Pass Vorschulkinder", in dem Präventionsmaßnahmen dokumentiert werden. Ein ef fektiver Ansatz der ECC-Prävention im Setting Kita ist das Programm „Kita mit Biss“, das 2003 in Frankfurt (Oder) initiiert wurde [43]. Mit der freiwilligen Selbstverpflichtung der Kitas, die Saugerflaschengabe $\mathrm{zu}$ unterbinden, ein zahngesundes Frühstück und regelmäßige Elternabende zur ECC-Prävention anzubieten, sowie mit der Unterstützung der Erzieher/-innen beim täglichen Zähneputzen wurde ein nachhaltiges gesundheitsförderndes Umfeld in den Kitas geschaffen.

\section{Fazit}

Ein niedriger Sozialstatus, das Alter und die nächtliche Gabe der Saugerflasche sind die stärksten Faktoren, die mit einer ECC bei 13 bis 36 Monate alten Kindern im Land Brandenburg assoziiert sind. Der Kariesbefall ist auf eine kleine Risikogruppe konzentriert. Gruppenprophylaktische Maßnahmen zur Reduktion der ECC-Prävalenz sind nur erfolgreich, wenn sie diese Risikogruppe erreichen. Zielführend dürfte nur eine intersektorale Zusammenarbeit der zuständigen ZÄD mit den Erzieher/-innen in Kitas, den Eltern, Zahnärzten und Kinderärzten sein. Settingorientierte Präventionsprogramme, die das tägliche unterstützende Zähneputzen mit einer fluoridhaltigen Kinderzahnpaste sowie den Verzicht auf die Gabe der Saugerflasche einschließen, sind flächendeckend im Land umzusetzen und durch eine begleitende Gesundheitsförderung mit Hebammen-, Schwangeren- und Elternschulungen zu flankieren. Aufgabe der ZÄD und/oder der niedergelassenen Zahnärzte ist es, Fluoridlacke risikoorientiert zu applizieren, um das Kariesrisiko der Kleinkinder zu reduzieren. Langfristig ist mit diesem intersektoralen Präventionsansatz zu erwarten, dass die gesundheitliche Benachteiligung von Kindern mit einem niedrigen sozialen Status kompensiert wird.

\section{Korrespondenzadresse}

\section{Prof. Dr. R. Heinrich-Weltzien}

Poliklinik für Präventive Zahnheilkunde und Kinderzahnheilkunde, Universitätsklinikum Jena

Bachstr.18, 07743 Jena

roswitha.heinrich-weltzien@med.uni-jena.de

Danksagungen. Die Autoren danken den Mitarbeiter/-innen der beteiligten ZÄD und dem Landesamt für Umwelt, Gesundheit und Verbraucherschutz (LUGV) für die Unterstützung bei der Durchführung dieser Studie. Das Projekt wurde von der Landeszahnärztekammer Brandenburg befürwortet.

Interessenkonflikt. Der korrespondierende Autor gibt für sich und seine Koautoren an, dass kein Interessenkonflikt besteht.

\section{Literatur}

1. American Academy of Pediatric Dentistry (AAPD) (2008) Definition of Early Childhood Caries (ECC). http://www.aapd.org/media/policies_guidelines/d_ecc.pdf

2. Azevedo TD, Bezerra AC, Toledo OA (2005) Feeding habits and severe early childhood caries in Brazilian preschool children. Pediatr Dent 27:2833

3. De Grauwe A, Aps JK, Martens LC (2004) Early Childhood Caries (ECC): What's in a name? Eur J Paediatr Dent 5:62-70

4. Declerck D, Leroy R, Martens L et al (2008) Factors associated with prevalence and severity of caries experience in preschool children. Community Dent Oral Epidemiol 36:168-178

5. Ferro R, Besostri A, Meneghetti B, Beghetto $M$ (2004) Comparison of data on Early Childhood Caries (ECC) with previous data for Baby Bottle Tooth Decay (BBTD) in an Italian kindergarten population. Eur J Paediatr Dent 5:71-75

6. Rosenblatt A, Zarzar P (2002) The prevalence of early childhood caries in 12- to 36-month-old children in Recife, Brazil. ASDC J Dent Child 69:319324

7. Kumarihamy SL, Subasinghe LD, Jayasekara P et al (2011) The prevalence of early childhood caries in 1-2 yrs olds in a semi-urban area of Sri Lanka. BMC Res Notes 4:336

8. Dye BA, Tan S, Smith V et al (2007) Trends in oral health status: United States, 1988-1994 and 1999-2004. Vital Health Stat 11:1-92

9. Splieth CH, Treuner A, Berndt C (2009) Orale Gesundheit im Kleinkindalter. Prävention Gesundheitsförderung 4:119-124

10. Feldens CA, Giugliani ER, Vigo A, Vitolo MR (2010) Early feeding practices and severe early childhood caries in four-year-old children from southern Brazil: a birth cohort study. Caries Res 44:445-452

11. Micheelis W, Schiffner U, Hoffmann T et al (2007) Ausgewählte Ergebnisse der Deutschen Mundgesundheitsstudie (DMS IV). Dtsch ZahnärztI Z 62:218-240
12. Finlayson $T L$, Siefert K, Ismail Al, Sohn W (2007) Psychosocial factors and early childhood caries among low-income African-American children in Detroit. Community Dent Oral Epidemiol 35:439448

13. Psoter WJ, Pendrys DG, Morse DE et al (2006) Associations of ethnicity/race and socioeconomic status with early childhood caries patterns. J Public Health Dent 66:23-29

14. Qin M, Li J, Zhang S, Ma W (2008) Risk factors for severe early childhood caries in children younger than 4 years old in Beijing, China. Pediatr Dent 30:122-128

15. Hallett KB, O'Rourke PK (2003) Social and behavioural determinants of early childhood caries. Aust Dent J 48:27-33

16. Warren JJ, Weber-Gasparoni K, Marshall TA et al (2008) Factors associated with dental caries experience in 1-year-old children. J Public Health Dent 68:70-75

17. Mohebbi SZ, Virtanen Jl, Vahid-Golpayegani M, Vehkalahti MM (2008) Feeding habits as determinants of early childhood caries in a population where prolonged breastfeeding is the norm. Community Dent Oral Epidemiol 36:363-369

18. Casamassimo PS, Thikkurissy S, Edelstein BL Maiorini E (2009) Beyond the dmft: the human and economic cost of early childhood caries. J Am Dent Assoc 140:650-657

19. Abanto J, Carvalho TS, Mendes FM et al (2011) Impact of oral diseases and disorders on oral healthrelated quality of life of preschool children. Community Dent Oral Epidemiol 39:105-114

20. Weintraub JA, Ramos-Gomez F, Jue B et al (2006) Fluoride varnish efficacy in preventing early childhood caries. J Dent Res 85:172-176

21. European Academy of Paediatric Dentistry (EAPD) (2008) Guidelines on prevention of Early Childhood Caries: an EAPD Policy Document. http:// www.eapd.gr/dat/1722F50D/file.pdf

22. Leroy R, Bogaerts K, Martens L, Declerck D (2012) Risk factors for caries incidence in a cohort of Flemish preschool children. Clin Oral Investig 16:805-812

23. Ammari JB, Baqain ZH, Ashley PF (2007) Effects of programs for prevention of early childhood caries. A systematic review. Med Princ Pract 16:437-442

24. American Academy of Pediatric Dentistry (AAPD) (2006) Proceedings of the symposium on the prevention of oral disease in children and adolescents 2005. Pediatr Dent 28:95-192

25. American Academy of Pediatric Dentistry (AAPD) (2011) Policy on Early Childhood Caries (ECC): classifications, consequences, and preventive strategies. http://www.aapd.org/media/Policies_ Guidelines/P_ECCClassifications.pdf

26. Böhm A, Ellsäßer G, Lüdecke K (2007) Der Brandenburger Sozialindex: Ein Werkzeug für die Gesundheits- und Sozialberichterstattung auf Landes- und kommunaler Ebene bei der Analyse von Einschülerdaten. Gesundheitswesen 69:555-559

27. World Health Organization (1997) Oral health surveys: Basic methods, 4. Aufl. World Health Organisation, Geneva

28. Brandenburger Ministerium für Umwelt, Gesundheit und Verbraucherschutz (MUGV) (2010) Leitfaden für Zahnärztliche Dienste der Gesundheitsämter im Land Brandenburg. http://www.gesundheitsplattform.brandenburg/Gesundheitsberichte

29. Klein H, Palmer CE, Knutson JW (1938) Studies on dental caries. Public Health Rep 53:47 
30. Bratthall D (2000) Introducing the significant caries index together with a proposal for a new global oral health goal for 12-year-olds. Int Dent J 50:378-384

31. Wyne AH (1999) Early childhood caries: nomenclature and case definition. Community Dent Oral Epidemiol 27:313-315

32. Baden A, Schiffner U (2008) Milchzahnkaries bei 3- bis 6-jährigen Kindern im Landkreis Steinburg. Oralprophylaxe Kinderzahnheilkd 30:70-74

33. Nies SM, Schauß SS, Siahi-Benlarbi R et al (2008) Häufigkeit und Typisierung der Milchzahnkaries bei Kindergartenkindern in Mittelhessen. Oralprophylaxe Kinderzahnheilkd 30:106-111

34. Pieper K, Jablonski-Momeni A (2008) Prävalenz der Milchzahnkaries in Deutschland. Oralprophylaxe Kinderzahnheilkd 30:6-10

35. Gussy MG, Waters EG, Walsh O, Kilpatrick NM (2006) Early childhood caries: current evidence for aetiology and prevention. J Paediatr Child Health 42:37-43
36. Leitlinie „Fluoridierungsmaßnahmen“. http:// www.dgzmk.de/uploads/tx_szdgzmkdocuments/ Fluoridierungsmassnahmen_Langversion.pdf

37. Twetman S (2008) Prevention of Early Childhood Caries (ECC) - review of literature published 1998-2007. Eur Arch Paediatr Dent 9:12-18

38. Marinho VC, Higgins JP, Sheiham A, Logan S (2004) Combinations of topical fluoride (toothpastes, mouthrinses, gels, varnishes) versus single topical fluoride for preventing dental caries in children and adolescents. Cochrane Database Syst Rev:CD002781

39. Plutzer K, Spencer AJ (2008) Efficacy of an oral health promotion intervention in the prevention of early childhood caries. Community Dent Oral Epidemiol 36:335-346

40. Neumann AS, Lee KJ, Gussy MG et al (2011) Impact of an oral health intervention on pre-school children $<3$ years of age in a rural setting in Australia. J Paediatr Child Health 47:367-372
41. § 26 Abs. 1 Satz 2 SGB V, Richtlinien des Bundesausschusses der Zahnärzte und Krankenkassen über die Früherkennungsuntersuchungen auf Zahn-, Mund- und Kieferkrankheiten. www.gkvspitzenverband.de/upload/Früherkennungs-RiLi_3644.pdf

42. Lee JY, Bouwens TJ, Savage MF, Vann WF Jr (2006) Examining the cost-effectiveness of early dental visits. Pediatr Dent 28:102-105

43. Haak P (2011) "Kita mit Biss"- Die Aktion wird Programm. https://www.frankfurt-oder.de/DE/ buergerservice/DezernateAemter/Soziales/Download\%20Zahnaerztlicher\%20Dienst/Evaluation\%20KitamitBiss\%20Schulj.\%202010\%202011. pdf

\section{Hier steht eine Anzeige.}

\section{Springer}




\section{$5 \quad$ Weiterführende Auswertungen und Ergebnisse}

Neben den in der vorliegenden Originalarbeit dargestellten Ergebnissen wurden weitere Auswertungen und statistische Analysen durchgeführt, die nachfolgend vorgestellt werden.

\subsection{Kariesprävalenz und -befall in den einzelnen Kreisen im Land}

\section{Brandenburg}

Durch die Angabe der Kreisnummern in den Befunddaten der Mundgesundheitsuntersuchung war eine kreisbezogene Analyse von Kariesprävalenz und -befall der 1-3-Jährigen möglich. Dabei wurden erhebliche Unterschiede zwischen den einzelnen Landkreisen und kreisfreien Städten offensichtlich (Tab. 4 und Abb. 2). So lag der Kariesbefall im Landkreis Elbe-Elster bei 1,0 \pm 2,4 dmft, wohingegen er im Landkreis Potsdam-Mittelmark 0,03 \pm 0,2 dmft betrug. Im Landkreis Dahme-Spreewald wurden hingegen bei den 57 untersuchten Kindern keine kariösen Läsionen festgestellt. Die Kariesprävalenz variierte ebenfalls zwischen 25\% (Landkreis Elbe-Elster) und 0\% (Landkreis Dahme-Spreewald). Bei der Betrachtung von Kindern mit niedrigem Sozialstatus in den einzelnen Kreisen (Abb. 3), wurden Parallelen zur Verteilung des Kariesbefalls in Relation zum Sozialstatus im Bundesland Brandenburg deutlich. Beispielsweise wiesen Kinder im Landkreis Elbe-Elster mit 1,0 $\pm 2,4$ dmft den höchsten Kariesbefall und mit 15,7\% den zweithöchsten Anteil von Kindern mit einem niedrigen Sozialstatus auf.

Durch die Beschränkung auf die teilnehmenden 8 Landkreise und 2 kreisfreien Städte und die Anonymisierung der einbezogenen Kitas war keine Unterscheidung in Berlin-Nahe und Berlin-Ferne Regionen möglich. Damit ließ sich nicht prüfen, ob das höhere Einkommensniveau, die höhere Bevölkerungs- und Kita-Dichte sowie der höhere Versorgungsgrad von Ärzten in Berlins „Speckgürtel“ einen positiven Einfluss auf die Karieserfahrung der dort lebenden Kinder hat (Berlin-Institut 2007).

Die Daten zur Mundgesundheit aus den einzelnen Kreisen zeigen weiterhin, dass die Polarisation des Kariesbefalls auf eine Kariesrisikogruppe ein landesweites Phänomen ist. So war der SiC-Index in fast allen Kindern aus den beteiligten Kreisen mehr als das 3-fache höher als der mittlere Kariesbefall der Population (Tab. 4). 
Tab. 4: Kariesprävalenz und -befall von 1-3-Jährigen in zehn Kreisen des Bundeslandes Brandenburg

\begin{tabular}{|c|c|c|c|c|c|c|c|}
\hline Kreis & $\begin{array}{c}\text { Anzahl } \\
\text { Kinder } \\
\text { (N) }\end{array}$ & $\begin{array}{l}\text { Anzahl } \\
\text { Zähne } \\
x \pm \text { SD }\end{array}$ & $\begin{array}{l}\text { Anzahl } \\
\text { gesunder } \\
\text { Zähne } \\
\text { x } \pm \text { SD }\end{array}$ & $\begin{array}{c}\begin{array}{c}\text { Karies- } \\
\text { prävalenz } \\
\text { d1/ d3 } \\
\text { Niveau (\%) }\end{array}\end{array}$ & $\begin{array}{l}\mathrm{dmft} \\
\mathrm{x} \pm \mathrm{SD}\end{array}$ & $\begin{array}{l}\text { d3-4mft } \\
x \pm S D\end{array}$ & $\begin{array}{c}\text { SiC (dmft) } \\
x \pm \text { SD }\end{array}$ \\
\hline BRB & 52 & $16,7 \pm 3,2$ & $16,4 \pm 3,3$ & $5,8 / 3,8$ & $0,3 \pm 1,6$ & $0,2 \pm 1,1$ & $0,9 \pm 2,8$ \\
\hline FF & 71 & $16,2 \pm 3,6$ & $15,9 \pm 3,6$ & $11,3 / 7,0$ & $0,3 \pm 1,0$ & $0,2 \pm 0,8$ & $1,0 \pm 1,7$ \\
\hline LDS & 57 & $17,9 \pm 2,7$ & $17,9 \pm 2,7$ & $0,0 / 0,0$ & $0,0 \pm 0,0$ & $0,0 \pm 0,0$ & $0,0 \pm 0,0$ \\
\hline EE & 52 & $18,7 \pm 2,0$ & $17,8 \pm 3,0$ & $25,0 / 19,2$ & $1,0 \pm 2,4$ & $0,8 \pm 2,1$ & $3,4 \pm 3,3$ \\
\hline HVL & 54 & $16,6 \pm 3,3$ & $16,3 \pm 3,4$ & $7,4 / 5,6$ & $0,3 \pm 1,3$ & $0,2 \pm 1,2$ & $0,9 \pm 2,3$ \\
\hline OSL & 67 & $16,7 \pm 4,0$ & $16,6 \pm 4,0$ & $6,0 / 3,0$ & $0,1 \pm 0,6$ & $0,1 \pm 0,5$ & $0,4 \pm 1,0$ \\
\hline OPR & 65 & $14,3 \pm 4,1$ & $14,2 \pm 4,1$ & $3,1 / 1,5$ & $0,1 \pm 0,6$ & $0,1 \pm 0,6$ & $0,3 \pm 1,1$ \\
\hline PM & 34 & $17,4 \pm 4,1$ & $17,3 \pm 4,1$ & $2,9 / 2,9$ & $0,03 \pm 0,2$ & $0,03 \pm 0,2$ & $0,1 \pm 0,3$ \\
\hline SPN & 156 & $16,3 \pm 4,1$ & $16,1 \pm 4,1$ & $7,7 / 5,8$ & $0,3 \pm 1,1$ & $0,2 \pm 1,0$ & $0,8 \pm 1,8$ \\
\hline UM & 53 & $15,7 \pm 4,7$ & $15,6 \pm 4,7$ & $3,8 / 3,8$ & $0,1 \pm 0,6$ & $0,1 \pm 0,6$ & $0,3 \pm 1,0$ \\
\hline
\end{tabular}




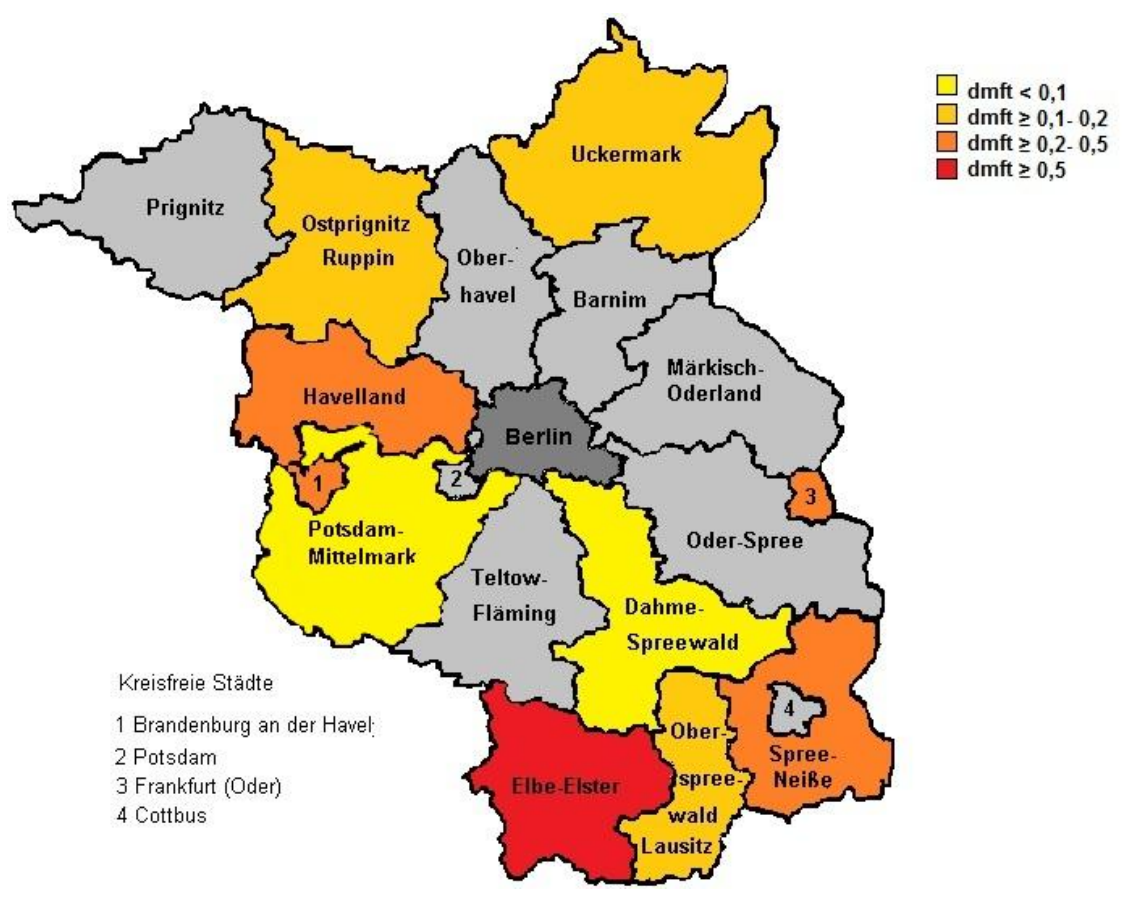

Abb. 2: Kreisbezogene Verteilung des Kariesbefall (Höhe der dmft-Werte) im Land Brandenburg

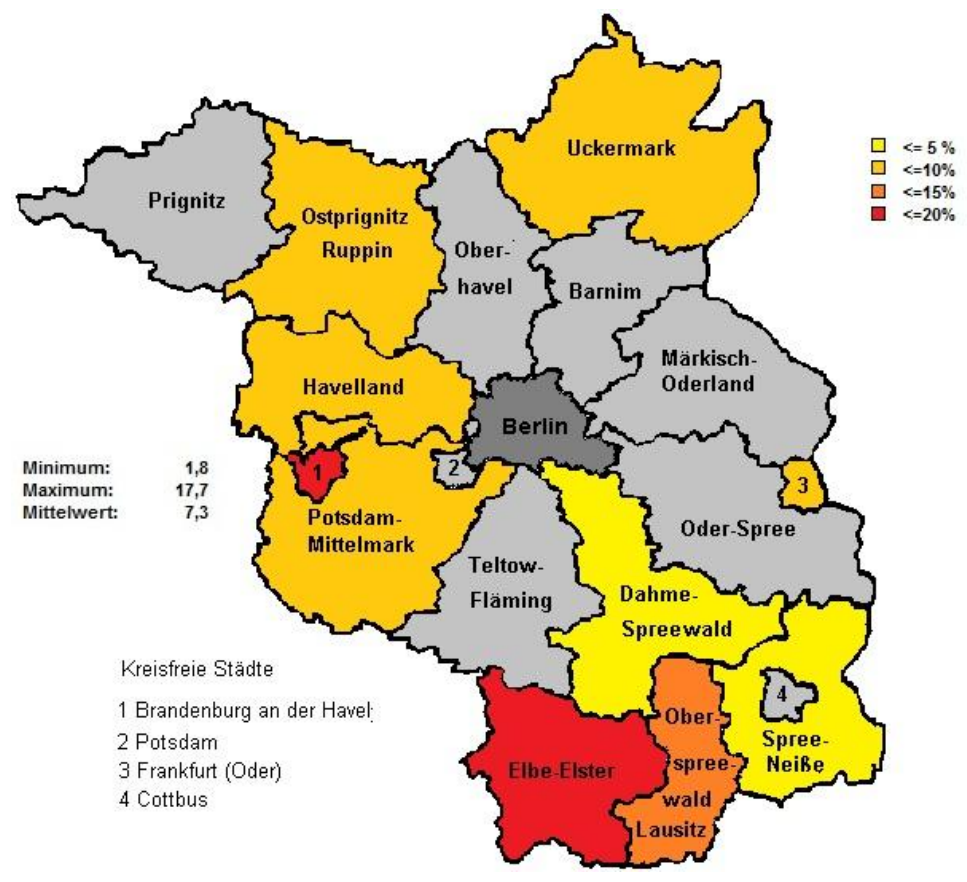

Abb. 3: Kreisbezogene Verteilung der Familien mit niedrigem Sozialstatus im Land Brandenburg 
5.2 Einfluss des Sozialstatus auf den Kariesbefall und das Mundgesundheitsverhalten bei Kleinkindern im Land Brandenburg

Die Beziehung zwischen dem Sozialstatus der Eltern und dem praktizierten Gesundheitsverhalten wurde anhand der Verteilung der fragebogenbasierten Antworten bivariat analysiert und mit dem Chi-Quadrat Test auf signifikante Unterschiede geprüft (Tab. 5).

Tab. 5: Beziehung zwischen Gesundheitsverhalten der Eltern und ihrem Sozialstatus

\begin{tabular}{|c|c|c|c|c|c|}
\hline & $\begin{array}{c}\text { Niedriger } \\
\text { Sozialstatus }\end{array}$ & $\begin{array}{c}\text { Mittlerer } \\
\text { Sozialstatus }\end{array}$ & $\begin{array}{c}\text { Hoher } \\
\text { Sozialstatus }\end{array}$ & Gesamt & $\begin{array}{c}\text { p- } \\
\text { Wert }\end{array}$ \\
\hline \multicolumn{6}{|c|}{ Seit wann putzt Ihr Kind die Zähne? } \\
\hline Seit 1. Zahn & 35,4 & 59,7 & 56,1 & 56,3 & \multirow{4}{*}{0,003} \\
\hline Seit 1. Lebensjahr & 47,9 & 33,7 & 34,6 & 35,1 & \\
\hline Seit 2. Lebensjahr & 12,5 & 6,3 & 9,0 & 8,0 & \\
\hline Noch gar nicht & 4,2 & 0,3 & 0,3 & 0,6 & \\
\hline \multicolumn{6}{|c|}{ Bekommt oder bekam Ihr Kind Fluoridtabletten? } \\
\hline Ja, bis zum 1. LJ & 37,5 & 29,8 & 29,0 & 30,0 & \multirow{4}{*}{0,020} \\
\hline Ja, bis zum 2. LJ & 37,5 & 42,8 & 48,3 & 45,0 & \\
\hline Ja, bis zum 3. LJ & 2,1 & 10,7 & 4,0 & 7,0 & \\
\hline Nein & 22,9 & 16,7 & 18,7 & 18,1 & \\
\hline \multicolumn{6}{|c|}{ Welches Speisesalz verwenden Sie im Haushalt? } \\
\hline Einfaches & 20,8 & 10,3 & 5,4 & 8,8 & \multirow{3}{*}{0,003} \\
\hline $\begin{array}{l}\text { mit Jod } \\
\text { angereichertes }\end{array}$ & 33,3 & 37,9 & 34,1 & 35,8 & \\
\hline $\begin{array}{l}\text { mit Jod und Fluorid } \\
\text { angereichertes }\end{array}$ & 45,8 & 51,8 & 60,5 & 55,4 & \\
\hline \multicolumn{6}{|c|}{ Wie lange wurde Ihr Kind gestillt? } \\
\hline Gar nicht & 25,0 & 20,1 & 14,3 & 17,8 & 0,008 \\
\hline
\end{tabular}




\begin{tabular}{|c|c|c|c|c|c|}
\hline 0-12 Monate & 75,0 & 75,7 & 76,4 & 76,0 & \\
\hline 13-24 Monate & 0,0 & 3,3 & 9,0 & 5,7 & \\
\hline 25-36 Monate & 0,0 & 1,0 & 0,3 & 0,6 & \\
\hline \multicolumn{6}{|c|}{ Wie lange bekam Ihr Kind die Flasche? } \\
\hline Gar nicht & 50,0 & 44,6 & 44,0 & 44,7 & \multirow{4}{*}{0,047} \\
\hline 0-12 Monate & 10,4 & 10,5 & 10,3 & 10,4 & \\
\hline 13-24 Monate & 18,8 & 27,9 & 35,7 & 30,8 & \\
\hline 25-36 Monate & 20,8 & 17,0 & 10,0 & 14,1 & \\
\hline \multicolumn{6}{|c|}{ Wie oft bekommt Ihr Kind zuckerreiche Getränke zwischen den Mahlzeiten? } \\
\hline Selten & 41,7 & 51,1 & 58,5 & 53,8 & \multirow{6}{*}{0,085} \\
\hline Bis zu 3-mal pro & & & & & \\
\hline Woche & 6,2 & 6,9 & 7,6 & 7,2 & \\
\hline einmal täglich & 18,8 & 14,1 & 15,6 & 15,1 & \\
\hline 2- bis 3-mal täglich & 18,8 & 16,4 & 13,0 & 15,0 & \\
\hline Öfter & 14,6 & 11,5 & 5,3 & 8,9 & \\
\hline \multicolumn{6}{|c|}{ Wie alt sind Sie? } \\
\hline Bis 20 Jahre & 8,3 & 3,3 & 0,3 & 2,3 & \multirow{5}{*}{0,001} \\
\hline Bis 25 Jahre & 33,3 & 16,8 & 7,7 & 13,8 & \\
\hline Bis 30 Jahre & 27,1 & 31,6 & 32,7 & 31,7 & \\
\hline Bis 35 Jahre & 18,8 & 30,3 & 40,7 & 34,2 & \\
\hline Über 35 Jahre & 12,5 & 18,1 & 18,7 & 17,9 & \\
\hline \multicolumn{6}{|c|}{ Wie viele Kinder leben in Ihrem Haushalt? } \\
\hline 1 & 39,6 & 48,9 & 57,3 & 52,1 & \multirow{2}{*}{0,001} \\
\hline 2 & 39,6 & 43,9 & 36,8 & 40,3 & \\
\hline
\end{tabular}




\begin{tabular}{|c|c|c|c|c|c|}
\hline 3 & 12,5 & 6,2 & 4,6 & 6,0 & \\
\cline { 1 - 5 } 4 und mehr & 8,3 & 1,0 & 1,3 & 1,7 & \\
\hline \multicolumn{5}{|c|}{ Wie viele Erwachsene leben in Ihrem Haushalt? } \\
\hline $\mathbf{1}$ & 43,8 & 16,7 & 6,3 & 13,9 & $\mathbf{0 , 0 0 1}$ \\
\hline $\mathbf{2}$ & 56,2 & 83,3 & 93,7 & 86,1 & \\
\hline
\end{tabular}

Es wurde deutlich, dass der Sozialstatus der Eltern einen hohen Einfluss auf gesundheitsrelevante Verhaltensweisen hat, bzw. das häusliche Umfeld des Kindes erheblich durch den Sozialstatus geprägt wird.

So putzten nur $35,4 \%$ der Eltern mit einem niedrigen Sozialstatus die Zähne ihres Kindes ab dem ersten Zahn, während dies in der Gesamtpopulation 56,3\% praktizierten. Signifikant war auch der Einfluss des Sozialstatus auf die Fluoridaufnahme des Kindes. Eltern mit niedrigem Sozialstatus gaben ihren Kindern seltener Fluoridtabletten und verwandten seltener fluoridhaltiges Speisesalz in ihrem Haushalt, als dies in der Gesamtpopulation der Fall war.

Es zeigte sich auch, dass Mütter mit niedrigem Sozialstatus ihr Kind seltener und kürzer stillten als Mütter mit einem mittleren oder hohen Sozialstatus. Erstere gaben weiterhin ihrem Kind mehr als doppelt so oft die Flasche bis über das zweite Lebensjahr hinaus, als Mütter mit einem hohen Sozialstatus.

Obwohl der Einfluss des Sozialstatus auf die Gabe von zuckerhaltigen Getränken zwischen den Hauptmahlzeiten nicht signifikant (Chi-Quadrat Test: $p=0,085$ ) war, zeigte sich beim Test linear-mit-linear, also einer Prüfung auf einen Trend für jede einzeln genommene Zeilen- und Spaltenvariable eine Signifikanz von $p=0,001$. So bekamen Kinder mit niedrigem Sozialstatus fast 3-mal so häufig „öfter als 2- bis 3mal täglich“ zuckerhaltige Getränke als Zwischenmahlzeiten, im Vergleich zu Kindern mit hohem Sozialstatus (14,6\% versus $5,3 \%)$.

Es konnte außerdem festgestellt werden, dass Eltern mit niedrigem Sozialstatus häufig jünger waren, mehr Kinder hatten und häufiger alleinerziehend waren als der Durchschnitt der Gesamtpopulation. 


\subsection{Ergebnisse der Elternbefragung zum Mundgesundheitsverhalten bei}

\section{ihren Kindern}

Aus den Antworten der 661 befragten Eltern im Land Brandenburg lässt sich ein gutes Abbild ihres Gesundheitsverhaltens generieren (Tab. 6). So besitzen fast alle Kinder (99,5\%) eine eigene Zahnbürste. Mehr als die Hälfte aller Eltern begannen bei Durchbruch des ersten Milchzahnes mit dem Putzen und fast $80 \%$ putzen mindestens 2-mal täglich die Zähne ihres Kindes. Weiterhin wird eine fluoridhaltige Kinderzahnpasta von den meisten Eltern verwandt $(88,7 \%)$ und auf die regelmäßige Verwendung von zuckerhaltigen Medikamenten wird weitestgehend verzichtet. In $55 \%$ der befragten Haushalte wird fluoridhaltiges Speisesalz verwendet und 81,1\% der Kinder bekamen in ihrem Leben schon Fluoridtabletten verabreicht. 82,2\% der Kinder wurden gestillt, davon 6,2\% noch über das erste Lebensjahr hinaus. Mehr als die Hälfte der Kinder (55,2\%) bekam die Flasche zum Trinken verabreicht, jedoch bekamen nur $14,4 \%$ diese auch während der Nacht. Von den Befragten waren nur 2,1\% der Eltern mit ihrem Kind bereits vor dem ersten Geburtstag beim Zahnarzt. Von den Eltern, die mit ihren Kindern bereits beim Zahnarzt waren, erhielten 42,8\% eine Aufklärung zur Vorbeugung der ECC und anderen für die Zahngesundheit des Kleinkindes relevanten Verhaltensweisen. Über 90\% der Kinder die bereits bei ihrem Hauszahnarzt waren, taten dies zu einer routinemäßigen Kontrolluntersuchung. 7,1\% der Eltern erhielten eine Aufforderung zum Zahnarztbesuch durch die ZÄD; 2,3\% der Eltern suchten auf Grund von Schmerzen den Hauszahnarzt auf. 38\% der Kinder nahmen mindestens einmal täglich zuckerhaltige Getränke zwischen den Hauptmahlzeiten zu sich.

Tab. 6: Antwortverteilung in der Elternbefragung zum Gesundheitsverhalten

\begin{tabular}{|l|r|r|}
\hline \multicolumn{1}{|c|}{ Fragebogenvariable } & \multicolumn{1}{c|}{ Anzahl } \\
\hline Besitzt Ihr Kind eine eigene Zahnbürste? & 656 & 99,5 \\
\hline ja & 3 & 0,5 \\
\hline nein & 371 & 56,4 \\
\hline Wann haben Sie begonnen Ihrem Kind die Zähne zu putzen? & 230 & 35,0 \\
\hline seit Durchbruch des 1. Milchzahnes & 53 & 8,1 \\
\hline im 1. Lebensjahr & 4 & 0,6 \\
\hline im 2. Lebensjahr & \\
\hline noch gar nicht & \\
\hline
\end{tabular}




\begin{tabular}{|c|c|c|}
\hline \multicolumn{3}{|c|}{ Putzt sich Ihr Kind schon selbstständig die Zähne? } \\
\hline ja & 428 & 65,1 \\
\hline nein & 229 & 34,9 \\
\hline \multicolumn{3}{|c|}{ Wann putzen Sie Ihrem Kind bzw. putzt sich Ihr Kind die Zähne? } \\
\hline morgens & 28 & 4,3 \\
\hline abends & 106 & 16,1 \\
\hline morgens und abends & 444 & 67,6 \\
\hline öfter & 79 & 12,0 \\
\hline \multicolumn{3}{|c|}{$\begin{array}{l}\text { Falls sich Ihr Kind schon selbstständig die Zähne putzt; wie oft putzen Sie die Zähne } \\
\text { Ihres Kindes nach? }\end{array}$} \\
\hline immer & 317 & 73,9 \\
\hline einmal täglich & 63 & 14,7 \\
\hline unregelmäßig & 41 & 9,5 \\
\hline nie & 8 & 1,9 \\
\hline \multicolumn{3}{|c|}{ Was für eine Zahnpasta benutzt Ihr Kind? } \\
\hline eine fluoridhaltige Kinderzahnpasta & 560 & 88,7 \\
\hline eine fluoridfreie Kinderzahnpasta & 57 & 9,0 \\
\hline eine Erwachsenenzahnpasta & 5 & 0,8 \\
\hline weiß nicht & 9 & 1,4 \\
\hline \multicolumn{3}{|c|}{ Bekommt oder bekam Ihr Kind Fluoridtabletten (Fluoretten, Zymafluor, etc.)? } \\
\hline ja, bis zum 1. Lebensjahr & 195 & 29,7 \\
\hline ja, bis zum 2. Lebensjahr & 293 & 44,6 \\
\hline ja, bis zum 3. Lebensjahr & 45 & 6,8 \\
\hline nein & 120 & 18,3 \\
\hline weiß nicht & 4 & 0,6 \\
\hline \multicolumn{3}{|c|}{ Welches Speisesalz verwenden Sie im Haushalt? } \\
\hline Einfaches & 60 & 9,2 \\
\hline mit Jod angereichertes & 234 & 35,8 \\
\hline mit Jod und Fluorid angereichertes & 360 & 55,0 \\
\hline \multicolumn{3}{|c|}{ Wie lange haben Sie Ihr Kind gestillt? } \\
\hline gar nicht & 117 & 17,8 \\
\hline ja, bis zum 1. Lebensjahr & 501 & 76,0 \\
\hline ja, bis zum 2. Lebensjahr & 37 & 5,6 \\
\hline ja, bis zum 3. Lebensjahr & 4 & 0,6 \\
\hline \multicolumn{3}{|l|}{ Bekommt Ihr Kind die Flasche? } \\
\hline nein & 295 & 44,8 \\
\hline ja, bis zum 1. Lebensjahr & 69 & 10,5 \\
\hline
\end{tabular}




\begin{tabular}{|c|c|c|}
\hline ja, bis zum 2. Lebensjahr & 201 & 30,5 \\
\hline ja, bis zum 3. Lebensjahr & 94 & 14,3 \\
\hline \multicolumn{3}{|l|}{ Bekommt Ihr Kind nachts die Flasche? } \\
\hline ja & 95 & 14,4 \\
\hline nein & 565 & 85,6 \\
\hline \multicolumn{3}{|l|}{ Waren Sie mit Ihrem Kind schon beim Zahnarzt? } \\
\hline nein & 395 & 59,8 \\
\hline ja, mit etwa 6 Monaten & 14 & 2,1 \\
\hline ja, nach dem 1. Geburtstag & 108 & 16,3 \\
\hline ja, nach dem 2. Geburtstag & 61 & 9,2 \\
\hline ja, ohne Zeitangabe & 83 & 12,6 \\
\hline \multicolumn{3}{|l|}{ Was war der Grund des ersten Zahnarztbesuches? } \\
\hline Kontrolle & 241 & 90,6 \\
\hline Aufforderung durch den Zahnärztlichen Dienst in Kita & 19 & 7,1 \\
\hline Schmerzen & 6 & 2,3 \\
\hline \multicolumn{3}{|c|}{$\begin{array}{l}\text { Welche Empfehlungen haben Sie dabei von Ihrem Zahnarzt erhalten? } \\
\text { (Mehrfachnennungen möglich) }\end{array}$} \\
\hline keine & 125 & 47,2 \\
\hline Aufklärung zur Nuckelflaschenkaries & 73 & 27,7 \\
\hline Verwendung einer fluoridhaltigen Kinderzahnpasta & 80 & 30,2 \\
\hline Nachputzen der Zähne durch die Eltern & 99 & 37,5 \\
\hline Ernährungsberatung & 44 & 16,7 \\
\hline Verwendung von Speisesalz mit Fluorid & 23 & 8,7 \\
\hline \multicolumn{3}{|c|}{ Wie oft erhält Ihr Kind zuckerreiche Getränke zwischen den Hauptmahlzeiten? } \\
\hline selten & 357 & 54,1 \\
\hline bis zu 3-mal in der Woche & 48 & 7,3 \\
\hline einmal täglich & 99 & 15 \\
\hline 2- bis 3-mal täglich & 98 & 14,8 \\
\hline öfter & 58 & 8,8 \\
\hline \multicolumn{3}{|c|}{$\begin{array}{l}\text { Bekommt Ihr Kind regelmäßig zuckerhaltige Medikamente? (z.B. Husten- oder } \\
\text { Antibiotikasaft) }\end{array}$} \\
\hline ja & 55 & 8,3 \\
\hline nein & 593 & 90,0 \\
\hline weiß nicht & 11 & 1,7 \\
\hline \multicolumn{3}{|l|}{ Wie alt sind Sie? } \\
\hline bis 20 Jahre & 15 & 0,3 \\
\hline bis 25 Jahre & 90 & 13,7 \\
\hline
\end{tabular}




\begin{tabular}{|l|r|r|}
\hline bis 30 Jahre & 209 & 31,9 \\
\hline bis 35 Jahre & 225 & 34,3 \\
\hline über 35 Jahre & 117 & 17,8 \\
\hline Wie viele Kinder leben in Ihrem Haushalt? & 342 & 51,8 \\
\hline 1 & 268 & 40,6 \\
\hline 2 & 39 & 5,9 \\
\hline 3 & 11 & 1,7 \\
\hline 4 und mehr & & \\
\hline Wie viele Erwachsene leben in Ihrem Haushalt? & 94 & 14,2 \\
\hline 1 & 566 & 85,8 \\
\hline 2 & & \\
\hline Einteilung in Brandenburger Sozialindex & 48 & 7,3 \\
\hline niedriger Sozialstatus & 305 & 46,6 \\
\hline mittlerer Sozialstatus & 302 & 46,1 \\
\hline hoher Sozialstatus & & \\
\hline
\end{tabular}

Die Differenz in der Anzahl aller Befragten $(n=661)$ ergibt sich durch verweigerte oder nicht zutreffende Antworten. 


\section{Diskussion}

In der vorliegenden Studie wurden erstmals Daten zur frühkindlichen Karies bei 0-3Jährigen in einem Bundesland erhoben und deren Zusammenhang mit dem Vorliegen von Risikofaktoren untersucht. Die Kariesprävalenz lag mit 7,4\% auf Schmelzkaries-(d1-4)-niveau niedriger als in der nationalen und internationalen Literatur angegebenen Vergleichswerten in dieser Altersgruppe (Tab. 2 und 3). Der Kariesbefall belief sich auf 0,3 $( \pm 1,2) \mathrm{d} 1-4 \mathrm{mft}$. Die in der Erhebung beobachtete niedrige Kariesprävalenz lässt sich einerseits durch die bereits praktizierten Präventionsprogrammen in Kitas im Land Brandenburg und andererseits durch das junge Alter der Kinder erklären.

Als Hauptrisikofaktoren für das Auftreten einer ECC wurde ein niedriger Sozialstatus, die Gabe der Saugerflasche in der Nacht und ein höheres Alter der Kinder ermittelt.

Auffallend war die starke Polarisation des Kariesbefalls auf eine kleine Risikogruppe. So vereinten $2 \%$ der Kinder $52 \%$ der Karies auf sich. Der SiC-Index des Drittels einer Population mit dem höchsten Kariesbefall war mit 0,8 $( \pm 2,0)$ d1-4mft mehr als doppelt so hoch wie in der Gesamtpopulation der 13-36 Monate alten Kinder im Land Brandenburg. Daneben zeigte sich, dass die Hauptlast des Kariesbefalls auf die dKomponente entfiel und mit einem niedrigen Sanierungsgrad $(19,9 \%)$ und geringen Füllungsindex (10\%) der Milchzähne assoziiert ist. Bei 4,7\% aller Kinder wurde eine Behandlungsbedürftigkeit aufgrund von kavitierten Läsionen und bei 2,1\% ein durch Initialläsionen begründeter Präventionsbedarf festgestellt.

Um den Sanierungsgrad zu erhöhen, muss die Zugängigkeit von Kleinkindern in die Zahnarztpraxis verbessert werden. Die von den gesetzlichen Krankenkassen finanzierten Früherkennungsuntersuchungen („FU 1-3“) im Alter vom 30.-72.Lebensmonat, die neben einer Einschätzung des Kariesrisikos auch eine Ernährungs- und Mundhygieneberatung sowie Empfehlungen zur Fluoridprophylaxe beinhalten, weisen im Bundesland Brandenburg jedoch nur eine Inanspruchnahmequote von $33,2 \%$ aus und liegen damit geringfügig über dem bundesweiten Durchschnitt von 31,5\% (Schäfer et al. 2012). Dass eine hohe Erreichbarkeit der Kinder in dieser Altersgruppe möglich ist, zeigen die Vorsorgeuntersuchungen („U-Untersuchungen“) der Pädiater. So liegt der Anteil der Kinder, die an allen Vorsorgeuntersuchungen U1 bis U8 (von der Geburt bis zum 48. 
Lebensmonat) im Land Brandenburg teilgenommen haben, bei $85,3 \%$, und der Anteil derjenigen, die alle Untersuchungen von der 4. Lebenswoche bis zum vollendeten ersten Lebensjahr (U3 bis U6) teilnahmen sogar bei 94,2\%. Auch die Untersuchungen zwischen dem ersten und vierten Geburtstag (U6 bis U8) weisen allesamt eine Inanspruchnahme von über 90\% auf (LUGV 2012).

In den Vorsorgeuntersuchungen U5 (im 6.-7.-Lebensmonat) und U6 im (10.-12. Lebensmonat) sollen Eltern zu Mundhygiene und zahngesunder Ernährung aufgeklärt werden, wobei jedoch unklar ist, wie dies seitens der Pädiater praktiziert wird. Die Mundgesundheit von Kleinkindern sollte dazu einen höheren Stellenwert in der Ausbildung von Kinderärzten und anderen an präventiven Maßnahmen beteiligten Medizinern erhalten, um das ECC-Risiko korrekt einschätzen und mundgesundheitsschädliche Verhaltensweisen frühzeitig vermeiden zu können (Bottenberg et al. 2008, Balaban et al. 2012). Um die Inanspruchnahme des Zahnarztbesuches im ersten Lebensjahr und die Bedeutung der Mundgesundheit zu fördern, sollten die zahnärztlichen Früherkennungsuntersuchungen in das Kinderuntersuchungsheft der pädiatrischen Vorsorgeuntersuchungen aufgenommen werden. Dies sollte durch ein Verweisungssystem an die Zahnärzte/-innen des ÖGD oder niedergelassene Kollegen beim Erkennen eines erhöhten Kariesrisikos durch den Pädiater ergänzt werden. Dass die ECC-Prävalenz reduziert werden kann, wenn Pädiater Kinder unter 3 Jahren mit einem erhöhten Kariesrisiko zum Zahnarzt verweisen, wurde in einer jüngsten Studie ausgewiesen (Long et al. 2012).

Andererseits wurde jedoch auch festgestellt, dass Eltern mit niedrigem Sozialstatus sowie Alleinerziehende, die niedrigste Inanspruchnahme bei Vorsorgeuntersuchungen aufweisen (Grossman et al. 1996, Navarro-Rubio et al. 1995, Lutz 1990). Das ist insofern problematisch, da dies vor allem Kinder der Kariesrisikogruppe betrifft. Um Kinder, die nicht an Früherkennungs- und Vorsorgeuntersuchungen teilnehmen, zu erreichen, müssen setting-basierte Präventionsmaßnahmen wie die Gruppenprophylaxe in Kitas flächendeckend umgesetzt werden. Dabei sollte sich das Prophylaxeteam der ZÄD im Rahmen der Gruppenprophylaxe auch auf eine Wissensvermittlung an die Eltern fokussieren. Kita-Erzieher sollten angehalten werden, mundgesundheitsförderliche Bedingungen, wie gesunde Ernährung oder das gemeinsame ritualisierte Zähneputzen mit fluoridhaltiger Zahnpasta, zu realisieren (DAJ 2012). Allerdings betrugen die Ausgaben der gesetzlichen 
Krankenversicherungen im Jahr 2011 für die Gruppenprophylaxe nur etwa 42 Millionen Euro im Vergleich zu etwa 455 Millionen Euro für individualprophylaktische Maßnahmen (IP 1 bis IP 5), die jedoch nicht die Gesamtheit aller Kinder erreichen (SVLFG 2012). Eine setting-basierte ECC-Prävention wird durch das Kinderförderungsgesetz (KiföG) zwangsläufig an Bedeutung gewinnen, da ab dem 1. August 2013 ein Rechtsanspruch auf einen Betreuungsplatz für alle Kinder vom vollendeten ersten bis zum vollendeten dritten Lebensjahr besteht.

Die Präventionsarbeit in den Kitas und bei Tagesmüttern/-vätern muss durch die Kooperation von verschiedenen Multiplikatoren wie Eltern, Erzieher/-innen und ZÄDs gestaltet werden. Dabei sollte ein Hauptaugenmerk auf die Zusammenarbeit zwischen Eltern und Kita-Mitarbeitern gelegt werden, um das Verhalten des Kindes nachhaltig positiv zu beeinflussen. Es sollte ein regelmäßiger Austausch stattfinden, und die Eltern sollten umfassend über die Maßnahmen in der Kita informiert werden, damit die Entwöhnung von der Saugerflasche, gesunde Ernährungskonzepte und das tägliche Zähneputzen auch häuslich praktiziert werden. Ebenso kommt es auf die Schaffung von positiven Vorbildfunktionen an. Beispielsweise lernen Kinder im Alter von 1-6 Jahren ihr Essverhalten nicht durch Ernährungswissen sondern vorrangig durch Imitation von Vorbildern, also den Eltern und Erziehern, (Ellrott 2007). Gleiches dürfte sich auch auf die Mundhygienegewohnheiten übertragen lassen. Daher sollten Eltern und Erzieher gemeinsam mit den Kindern ihre Zähne putzen. Diese Maßnahmen ließen sich durch eine Selbstverpflichtung der Erzieher/innen und Kita-Träger, bestimmte Handlungsleitlinien konsequent im täglichen Betreuungsablauf in der Kita umzusetzen, realisieren. Solche Handlungsrichtlinien wurden in dem seit 2003 eingeführtem Programm „Kita mit Biss“ in Frankfurt/Oder praktiziert (Haak 2007). In diesen Kitas wird durch den Verzicht auf gesüßte Getränke, die Verabreichung von Obst als Zwischenmahlzeiten, die Abgewöhnung von Lutschgewohnheiten, den weitestgehenden Verzicht auf die Saugerflasche und das regelmäßige Zähneputzen nach jeder Hauptmahlzeit, ein mundgesundheitsförderndes Umfeld geschaffen (Haak 2011).

Diese täglichen Präventionsmaßnahmen sollten durch die ZÄD durch zusätzliche gruppenprophylaktische Maßnahmen ergänzt werden. Hierbei sind neben der Mundgesundheitsaufklärung und Kariesrisikoeinschätzung vor allem Fluoridierungsmaßnahmen entscheidend. Die Fluoridlackapplikation bei allen Kindern ab dem 6 . 
Lebensmonat ist zwar eine der teuersten präventiven Maßnahmen, resultiert aber in einer Kariesreduktion im Milchgebiss von 33\% (Marinho et al. 2002). Nach Strippel (2002) belaufen sich die Kosten einer professionellen Basis-Gruppenprophylaxe mit zweimal jährlicher Mundgesundheitserziehung und Fluoridlackapplikation in den Kitas bzw. Schulen auf etwa $16 €$ pro Kind und Jahr. 55\% der Ausgaben für die Fluoridlackapplikation dürften jedoch durch geringere Ausgaben für restaurative und konservierende Maßnahmen wieder eingespart werden (Hirsch et al. 2012). Weitaus niedrigere Kosten würden bei der Konzentration der Fluoridierungsmaßnahmen auf Kinder mit erhöhtem Kariesrisiko anfallen (Hirsch et al. 2012).

Neben der Verfügbarkeit von Fluoriden trägt die frühzeitige Aufklärung von Schwangeren und jungen Müttern wesentlich zur Prävention der ECC bei (Ammari et al. 2007). Dazu sollten Gynäkologen und Hebammen in ein intersektorales Präventionsprogramm einbezogen werden.

Die begleitende Gesundheitsfrühförderung und Aufklärung („anticipatory guidance“) junger Mütter in mündlicher und schriftlicher Form führt zu einer signifikanten Verbesserung der Mundgesundheit ihrer Kinder (Plutzer und Spencer 2008). Zum gleichen Ergebnis kommt auch die Arbeitsgruppe von Neumann et al. (2011), die neben der Gesundheitsförderung der Mütter, Zahnbürsten und Zahnpasta für ihre 7 und 8 Monate alten Kinder bereitstellten. Die Autoren zeigen jedoch auch, dass nur ein kontinuierlicher Kontakt zwischen zahnmedizinischem Personal und Eltern Erfolg versprechend ist, da nach 3-jähriger Beobachtungszeit keine Unterschiede in der ECC-Prävalenz zwischen der Interventions- und Kontrollgruppe beobachtet wurden, während sie sich im ersten und zweiten Untersuchungsjahr signifikant unterschieden. Der gesundheitsfördernde Effekt einer früh einsetzenden ECC-Prävention wurde auch in einem kommunalen Präventionsansatz in Form der Wöchnerinnenberatungen in Vorarlberg, Österreich, ausgewiesen (Wagner et al. 2011). 5-Jährige, deren Mütter in den kommunalen Krankenhäusern des Landes eine umfassende gesundheitliche Beratung zur Vorbeugung der ECC erhielten, wiesen eine signifikant bessere Mundgesundheit auf, als Kinder von Müttern ohne eine Gesundheitsberatung. Dieser positive Einfluss wurde auch bei Kindern mit einem Migrationshintergrund belegt, wenn gleich die sozial bedingte gesundheitliche Benachteiligung nicht vollständig kompensiert werden konnte. Diese Ergebnisse 
zeigen, dass eine frühzeitige Intervention Erfolg hat, wenn sie durch eine kontinuierliche und risikoorientierte zahnärztliche Betreuung fortgeführt wird.

In der Bundesrepublik Deutschland bietet das seit Beginn des Jahres 2012 in Kraft getretene „Gesetz zur Stärkung eines aktiven Schutzes von Kindern und Jugendlichen", auch „Bundeskinderschutzgesetz“ genannt, neue Möglichkeiten, dass werdende und junge Eltern über die örtlichen Leistungsangebote zur Beratung in Fragen der Schwangerschaft, Entwicklung und Gesundheit des Kindes, Informationen erhalten. Damit Interessenten schnelleren und leichteren Zugang zu Beratungsstellen erhalten, sind verbindliche Netzwerkstrukturen aufzubauen in die neben Institutionen des ÖGD auch Hebammen, niedergelassene Ärzte und Zahnärzte einbezogen werden (Berg 2012).

Ein weiterer wichtiger Ansatz, um die sozialen Schichten zu erreichen, die selten Präventionsangebote in Anspruch nehmen, sind aufsuchende Konzepte wie das Projekt „Familienhebammen" in Sachsen-Anhalt, das sich speziell an sozial benachteiligte Familien wendet (BZgA 2011). Die Betreuung der werdenden Mütter und Väter wird von speziell weitergebildeten Hebammen bis zum Ende des ersten Lebensjahres des Kindes in bis zu zehn Wochenstunden pro Familie durchgeführt. Neben der Beratung und Aufklärung in gesundheitsspezifischen Fragen kann durch die Familienhebamme auch die Förderung der Inanspruchnahme der Früherkennungs- und Vorsorgeuntersuchungen erreicht werden (BZgA 2011). Im Bundesland Brandenburg bestehen seit 2006 die regionalen „Netzwerke Gesunde Kinder" in denen ehrenamtliche Familienpaten in Kooperationsvereinbarungen mit Hebammen, Gynäkologen, Pädiatern, Schwangerenberatungsstellen und dem Jugend-, Sozial- und Gesundheitsamt zusammenarbeiten, um Familien im Bedarfsfall während der Schwangerschaft und in den ersten drei Lebensjahren des Kindes zu beraten und zu begleiten. Dazu erhalten die Familienpaten spezielle Schulungen, die auch die Prävention der ECC beinhalten, so dass dieses Wissen direkt an die Eltern weitervermittelt werden kann (BVPG 2012).

Diese aufsuchenden Angebote sowie die von verschiedenen Trägern angebotenen Elternschulungen oder Eltern-Kind-Gruppen sind besonders wichtig, um die Kinder zu erreichen, die keine Kita besuchen und somit keinen Zugang zu den gruppenprophylaktischen Maßnahmen gemäß § 21 SGB V finden. 
Die hohe Erfolgsquote der aufsuchenden Angebote, Elternschulungen oder Wöchnerinnenberatungen begründet sich auch darin, dass die Aufklärung und Wissensvermittlung im direkten, persönlichen Gespräch erfolgt. Dies wird von den meisten Eltern gegenüber dem bloßen Einsatz von Broschüren, Postern oder sonstigen Drucksachen bevorzugt und ist somit weitaus effektiver (Bergmann et al. 1998).

Um gerade sozial benachteiligte Familien in Präventionsprogramme einzubeziehen, sollten die Angebote kostenlos, mit freiwilliger Teilnahme, möglichst anonym und ohne größere Anmeldemodalitäten (die Abschreckungspotential haben könnten), gestaltet sein (BZgA 2011).

\section{Diskussion der Methodik}

Die Mundgesundheitsuntersuchung wurde von kalibrierten Zahnärztinnen der ZÄD unter Berücksichtigung der WHO-Kriterien (1997) und nach den Richtlinien des Leitfadens des Brandenburgischen Gesundheitsministeriums (MASGF 2009) durchgeführt, so dass von einer hohen Qualität und Vergleichbarkeit der Daten auszugehen ist. Aufgrund der visuell-taktilen Kariesdiagnostik könnte jedoch eine höhere Kariesprävalenz bei den 13-36 Monate alten Kindern vorliegen, da im Rahmen der Reihenuntersuchung ohne Trocknung der Zahnoberfläche und die Verwendung ergänzender diagnostischer Verfahren die approximal kariösen Läsionen unterschätzt werden (Poorterman et al. 2000). Wünschenswert wäre weiterhin eine Erhebung des zahnflächenbezogenen Kariesbefalls gewesen, da die Einschätzung des Kariesrisikos der Kinder in Verbindung mit den erfassten initialkariösen Läsionen den Präventionsbedarf genauer abbildet.

Da sich die ZÄD der entsprechenden Landkreise und kreisfreien Städte freiwillig zur Teilnahme am Kooperationsprojekt verpflichteten, kann nicht beurteilt werden, ob die Nichtteilnahme der übrigen Kreise einen relevanten Einfluss auf die ermittelten Ergebnisse hat. Es kann ebenso nicht ausgeschlossen werden, dass durch die unterschiedliche Anzahl untersuchter Kinder, die nicht immer in Relation zur absoluten Einwohnerzahl des jeweiligen Kreises standen, eine Über- bzw. Untergewichtung einzelner Kreise bezüglich der Gesamtkariesprävalenz eingetreten ist. Diese methodischen Limitationen dürften jedoch vor dem Hintergrund, dass es weder signifikante Unterschiede in der Alters- und Geschlechterverteilung, der Kariesprävalenz und dem Kariesbefall zwischen der Stichprobe mit Elternbefragung 
und der Gesamtpopulation aus den 10 Kreisen und kreisfreien Städten gab, unbedeutend sein. Retrospektiv betrachtet, wären vom Autor kleinere Änderungen am Fragebogendesign vorgenommen worden, um die Antwortmöglichkeiten, die in Abhängigkeit zum Alter des Kindes stehen, zu standardisieren (beispielsweise Flaschengabe „bis jetzt“ ohne genaue zeitliche Angabe). Da das Alter des jeweiligen Kindes zum Zeitpunkt der Untersuchung jedoch durch die anonymisierte Zuordnung der Befunddaten bekannt war, ließ sich dies in der Statistik anpassen. Zudem wurde die Beziehung zwischen dem Kariesbefall und einzelnen Fragebogenvariablen altersund geschlechtsadjustiert vorgenommen, um Verzerrungen der Ergebnisse zu vermeiden.

Aus den Antworten des Fragebogens konnte keine genaue Fluoridanamnese der Kinder erhoben werden. Es wurde zwar die Verwendung einer fluoridhaltigen Zahnpasta, von fluoridhaltigem Speisesalz und Fluoridtabletten erfragt, jedoch keine weiteren Daten zur Frequenz und Menge der Fluoridaufnahme erhoben, so dass keine Einschätzung der täglichen Fluorid-Gesamtzufuhr (in mg) möglich war.

In einigen Untersuchungen wird der Migrationshintergrund des Kindes als Risikofaktor für die ECC aufgezeigt (Hallett und O'Rourke 2003, Psoter et al. 2006). Dieser Aspekt wurde in der vorliegenden Studie nicht beleuchtet. Da der Ausländeranteil im Bundesland Brandenburg lediglich 2,8\% (BaMF 2012) und der Anteil von Menschen mit Migrationshintergrund nur 5,2\% (AfSBB 2012) betragen, ist konnte dieser Parameter in der vorliegenden Untersuchung zu vernachlässigen.

Ein Nachteil der Datenerhebung mit Fragebögen besteht in der fehlenden Überprüfbarkeit der gegebenen Antworten. Durch die Anonymisierung der Daten kann jedoch von einem höheren Wahrheitsgehalt der Antworten als bei einem personalisiertem Fragebogen ausgegangen werden, da die Antworten oftmals kritischer ausfallen (Hoeth et al. 1967). Einige Eltern lehnten allerdings eine Beantwortung der Fragen zum sozioökonomischen Hintergrund ab, so dass diese Fragebögen bei der Auswertung der jeweiligen Items nicht zur Verfügung standen. 


\section{Schlussfolgerungen}

Mit der vorliegenden Untersuchung konnte gezeigt werden, dass die Hauptrisikofaktoren für 13-36 Monate alte Kinder, die im Bundesland Brandenburg eine Kita besuchen oder von Tagesmüttern/-vätern betreut werden, an frühkindlicher Karies zu erkranken, ein Elternhaus mit niedrigem Sozialstatus, die nächtliche Gabe der Saugerflasche und ein höheres Alter der Kinder selbst, sind.

Des Weiteren konnten erstmals in dieser Altersgruppe bundeslandweite Daten zur Kariesprävalenz (7,4\%) und zum Kariesbefall (0,3 d1-4mft) gewonnen werden. Die starke Polarisation des Kariesbefalls und der niedrige Sanierungsgrad verdeutlichen die Notwendigkeit einer verstärkten präventiven Intervention, um die Kariesrisikogruppe zu erreichen. Dazu sind im Setting Kita Präventionsprogramme ritualisiert umzusetzen, die neben den Erziehern und Eltern auch die ZÄD einbeziehen und neben der Entwöhnung von der Saugerflasche vorrangig auf das tägliche supervisierte Zähneputzen mit fluoridhaltiger Kinderzahnpasta abzielen.

Darüber hinaus sollten Angebote für Kinder mit niedrigem Sozialstatus, die keine Kita besuchen, geschaffen werden. Dies kann durch frühestmögliche Aufklärung der Eltern durch Gynäkologen, Pädiater, Hebammen oder durch eine Wöchnerinnenberatung erfolgen, die durch eine begleitende Gesundheitsfrühförderung in Form einer aufsuchenden Betreuung ergänzt werden.

Um die unzureichende zahnärztliche Versorgung von Kleinkindern zu verbessern und einen frühzeitigen Zugang der Kinder in die Zahnarztpraxen zu erreichen, müssen Anreize für einen Zahnarztbesuch innerhalb der ersten zwei Lebensjahre geschaffen werden. Dazu könnte ein verbessertes Verweisungssystem der Pädiater an die Zahnärzte sowie die Aufnahme der zahnärztlichen Früherkennungsuntersuchungen in das Untersuchungsheft der pädiatrischen Vorsorgeuntersuchungen beitragen.

Durch früh beginnende, auf die Risikofaktoren der ECC abzielende und speziell die Risikogruppe adressierende Präventionsprogramme, kann die Prävalenz der frühkindlichen Karies zukünftig dauerhaft gesenkt und eine sozial bedingte gesundheitliche Benachteiligung kompensiert werden. 


\section{Literaturverzeichnis}

Abanto J, Carvalho TS, Mendes FM, Wanderley MT, Bonecker M, Raggio DP. 2011. Impact of oral diseases and disorders on oral health-related quality of life of preschool children. Community Dent Oral Epidemiol, 39 (2):105-114.

Al-Malik MI, Holt RD, Bedi R. 2001. The relationship between erosion, caries and rampant caries and dietary habits in preschool children in Saudi Arabia. Int $\mathrm{J}$ Paediatr Dent, 11 (6):430-439.

al-Shalan TA, Erickson PR, Hardie NA. 1997. Primary incisor decay before age 4 as a risk factor for future dental caries. Pediatr Dent, 19 (1):37-41.

American Academy of Pediatric Dentistry (AAPD). 2012. Guideline on Infant Oral Health Care. Pediatr. Dent, 34 (5):148-152.

American Academy of Pediatric Dentistry (AAPD). 2011. Policy on early childhood caries (ECC): classifications, consequences, and preventive strategies http://www.aapd.org/media/Policies_Guidelines/P_ECCClassifications.pdf.

Ammari JB, Baqain ZH, Ashley PF. 2007. Effects of programs for prevention of early childhood caries. A systematic review. Med Princ Pract, 16 (6):437-442.

Amt für Statistik Berlin-Brandenburg (AfSBB). 2012. Mikrozensus 2011 http://www.statistik-berlin.de/pms/2012/12-09-19b.pdf.

Azevedo TD, Bezerra AC, de Toledo OA. 2005. Feeding habits and severe early childhood caries in Brazilian preschool children. Pediatr Dent, 27 (1):28-33.

Baden A, Schiffner U. 2008. Milchzahnkaries bei 3- bis 6-jährigen Kindern im Landkreis Steinburg. Oralprophylaxe \& Kinderzahnheilkunde, 30:70-74. Balaban R, Aguiar CM, da Silva Araujo AC, Dias Filho EB. 2012. Knowledge of paediatricians regarding child oral health. Int J Paediatr Dent, 22 (4):286-291.

Berg B. 2012. Kindliche Gesundheit besser schützen. Zahnärztliche Mitteilungen, 102 (12).

Bergmann KE, Bergmann RL, Kamtsiuris P, Schoppa A, Huber M, Schulze S, Kahl H, Dörries A. 1998. Krankheitsprävention und Gesundheitsförderung in der Familie. Berlin: Robert-Koch-Institut.

Berkowitz RJ. 2003. Causes, treatment and prevention of early childhood caries: a microbiologic perspective. J Can Dent Assoc, 69 (5):304-307. 
Berlin-Institut. 2007. Gutachten zum demografischen Wandel im Land Brandenburg http://www.berlininstitut.org/fileadmin/user_upload/Studien/Brandenburg_Webversion.pdf.

Berlutti F, Catizone A, Ricci G, Frioni A, Natalizi T, Valenti P, Polimeni A. 2010. Streptococcus mutans and Streptococcus sobrinus are able to adhere and invade human gingival fibroblast cell line. Int J Immunopathol Pharmacol, 23 (4):1253-1260.

Berndt C, Splieth C. 2009. Zahnärztlicher Gesundheitsbericht 2008/09 http://www.greifswald.de/uploads/media/2008-2009Zahnaeztlicher_Gesundheitsbericht_.pdf.

Blumenshine SL, Vann WF, Gizlice Z, Lee JY. 2008. Children's school performance: impact of general and oral health. J Public Health Dent, 68 (2):82-87.

Boemanns B, Lorbeer J, Wetzel WE. 1997. Häufigkeit der Milchzahnkaries bei Kleinkindern. Oralprophylaxe, 19:133-139.

Born C, Brauns U, Dürr KG, Hartmann T, Panthen C, Schmidt-Schäfer S, VölknerStetefeld P, von der Burg C, Winter-Borucki G, Wleklinski C. 2005. Mundgesundheit 3- bis 5-jähriger Kindergartenkinder. Dtsch Hessische Z, 11:470-472.

Borutta A, Kneist S, Kischka P, Eherler D, Chemnitius P, Stösser L. 2002. Die Mundgesundheit von Kleinkindern in Beziehung zu relevanten Einflussfaktoren. Dtsch Zahnärztl Z, 57:682-687.

Bottenberg , Van Melckebeke L, Louckx F, Vandenplas Y. 2008. Knowledge of Flemish paediatricians about children's oral health--results of a survey. Acta Paediatr, 97 (7):959-963.

Brandenburger Landesamt für Umwelt Gesundheit und Verbraucherschutz (LUGV). 2012. Inanspruchnahme von Früherkennungsuntersuchungen bei Einschülern, Datenstand 2011.

Brandenburger Ministerium für Arbeit Soziales Gesundheit und Familie (MASGF). 2009. Leitfaden zur standardisierten Befunderhebung und Dokumentation zahnmedizinischer Angaben im Öffentlichen Gesundheitsdienst http://www.landkreis-oder-spree.de/media/custom/1300_24644_1.PDF.

British Association for the Study of Community Dentistry (BASCD), Department of Health (DH). 2009. Delivering Better Oral Health: An evidence- based toolkit for prevention 
http://www.dh.gov.uk/prod_consum_dh/groups/dh_digitalassets/documents/di gitalasset/dh_102982.pdf.

Bundesamt für Migration und Flüchtlinge (BaMF). 2012. Das Bundesamt in Zahlen 2011; Asyl, Migration, ausländische Bevölkerung und Integration http://www.bamf.de/SharedDocs/Anlagen/DE/Publikationen/Broschueren/bund esamt-in-zahlen-2011.pdf?_blob=publicationFile.

Bundesvereinigung Prävention und Gesundheitsförderung e.V. (BVPG). 2012.

Dokumentation der Statuskonferenz 2011 "Frühkindliche

Gesundheitsförderung"

http://www.bvpraevention.de/bvpg/images/publikationen/bvpg_statusbericht_6 _web.pdf.

Bundeszentrale für Gesundheitliche Aufklärung (BZgA). 2011. Kriterien guter Praxis in der Gesundheitsförderung bei sozial Benachteiligten : Ansatz - Beispiele weiterführende Informationen. 5. Aufl. Köln: BZgA.

Carvalho JC, D'Hoore W, Van Nieuwenhuysen JP. 2004. Caries decline in the primary dentition of Belgian children over 15 years. Community Dent Oral Epidemiol, 32 (4):277-282.

Casamassimo PS, Thikkurissy S, Edelstein BL, Maiorini E. 2009. Beyond the dmft: the human and economic cost of early childhood caries. J Am Dent Assoc, 140 (6):650-657.

Caufield PW, Li Y, Bromage TG. 2012. Hypoplasia-associated severe early childhood caries--a proposed definition. J Dent Res, 91 (6):544-550.

De Grauwe A, Aps JK, Martens LC. 2004. Early Childhood Caries (ECC): what's in a name? Eur J Paediatr Dent, 5 (2):62-70.

Declerck D, Leroy R, Martens L, Lesaffre E, Garcia-Zattera MJ, Vanden Broucke S, Debyser M, Hoppenbrouwers K. 2008. Factors associated with prevalence and severity of caries experience in preschool children. Community Dent Oral Epidemiol, 36 (2):168-178.

Deutsche Arbeitsgemeinschaft für Jugendzahnpflege e.V. (DAJ). 2012. Frühkindliche Karies: Konsensfindung zu den zentralen Inhalten der Gruppenprophylaxe für unter 3-Jährige http://www.jugendzahnpflege.hzn.de/lagh_aktuell/I00C2C673.0/DAJEmpfehlu ngenU3_neu.pdf. 
Dye BA, Tan S, Smith V, Lewis BG, Barker LK, Thornton-Evans G, Eke PI, BeltranAguilar ED, Horowitz AM, Li CH. 2007. Trends in oral health status: United States, 1988-1994 and 1999-2004. Vital Health Stat 11, (248):1-92.

Ellrott T. 2007. Wie Kinder essen lernen. Ernaehr Wiss Prax, 1:167-173.

European Academy of Paediatric Dentistry (EAPD). 2008. Guidelines on Prevention of Early Childhood Caries: An EAPD Policy Document http://www.eapd.gr/dat/1722F50D/file.pdf.

Fass E. 1962. Is bottle feeding of milk a factor in dental caries? J Dent Child, 29:245251.

Feldens CA, Giugliani ER, Vigo A, Vitolo MR. 2010. Early feeding practices and severe early childhood caries in four-year-old children from southern Brazil: a birth cohort study. Caries Res, 44 (5):445-452.

Ferro R, Besostri A, Meneghetti B, Beghetto M. 2004. Comparison of data on Early Childhood Caries (ECC) with previous data for Baby Bottle Tooth Decay (BBTD) in an Italian kindergarten population. Eur J Paediatr Dent, 5 (2):71-75.

Finlayson TL, Siefert K, Ismail AI, Sohn W. 2007. Psychosocial factors and early childhood caries among low-income African-American children in Detroit. Community Dent Oral Epidemiol, 35 (6):439-448.

Fisher J, Johnston S, Hewson N, van Dijk W, Reich E, Eisele JL, Bourgeois D. 2012. FDI Global Caries Initiative; implementing a paradigm shift in dental practice and the global policy context. Int Dent J, 62 (4):169-174.

Gräßler G, Irmisch B, Viergutz G, Deketh M, Wilczek S, Hetzer G. 1998. Studie zur zahnmedizinischen Prophylaxe bei Dresdner Kleinkindern. Dtsch Zahnärztl Z, 53 (11):805-809.

Grossman LK, Humbert AJ, Powell M. 1996. Continuity of care between obstetrical and pediatric preventive care: indicators of nonattendance at the first well-child appointment. Clin Pediatr (Phila), 35 (11):563-569.

Gülzow H-J, Farshi H. 2000. Die Zahngesundheit Hamburger Kindergartenkinder 1977-1998. Dtsch Zahnärztl Z, 55 (11):770-773.

Gussy MG, Waters EG, Walsh O, Kilpatrick NM. 2006. Early childhood caries: current evidence for aetiology and prevention. J Paediatr Child Health, 42 (1-2):37-43.

Haak P. 2007. „Kita mit Biss“- Ein Präventionsprojekt zur Förderung der Mundgesundheit in den Kindertagesstätten der Stadt Frankfurt (Oder) http://www.gesundheitberlin.de/download/Haak,_P..pdf. 
Haak P. 2011. „Kita mit Biss“- Die Aktion wird Programm https://www.frankfurtoder.de/DE/buergerservice/DezernateAemter/Soziales/Download\%20Zahnrztli cher\%20Dienst/Evaluation\%20KitamitBiss\%20Schulj.\%202010\%202011.pdf.

Hallett KB, O'Rourke PK. 2003. Social and behavioural determinants of early childhood caries. Aust Dent J, 48 (1):27-33.

Haugejorden O, Birkeland JM. 2002. Evidence for reversal of the caries decline among Norwegian children. Int J Paediatr Dent, 12 (5):306-315.

Hellwig E, Klimek J, Attin T. 2009. Einführung in die Zahnerhaltung: Prüfungswissen Kariologie, Endodontologie und Parodontologie. Köln: Deutscher Ärzte-Verlag.

Hellwig E, Altenburger M, Attin T, Lussi A, Buchalla W. 2010. Remineralization of initial carious lesions in deciduous enamel after application of dentifrices of different fluoride concentrations. Clin Oral Investig, 14 (3):265-269.

Hirsch GB, Edelstein BL, Frosh M, Anselmo T. 2012. A simulation model for designing effective interventions in early childhood caries. Prev Chronic Dis, 9:66.

Hoeth F, Büttel R, Feyerabend H. 1967. Experimentelle Untersuchungen zur Validität von Persönlichkeitsfragebogen. Psychologische Rundschau, 18:169-184.

Horowitz HS. 1998a. Research issues in early childhood caries. Community Dent Oral Epidemiol, 26 (1):67-81.

Horowitz HS. 1998b. Research issues in early childhood caries. Community Dent Oral Epidemiol, 26 (1 Suppl):67-81.

Ismail AI, Sohn W. 1999. A systematic review of clinical diagnostic criteria of early childhood caries. J Public Health Dent, 59 (3):171-191.

Jacobi A. 1862. Dentition and its derangements: a course of lectures delivered in the New York Medical College. New York.

Kalyvas DI, Taylor CM, Michas V, Lygidakis NA. 2006. Dental health of 5-year-old children and parents' perceptions for oral health in the prefectures of Athens and Piraeus in the Attica County of Greece. Int J Paediatr Dent, 16 (5):352357.

Kaste LM, Gift HC. 1995. Inappropriate infant bottle feeding. Status of the Healthy People 2000 objective. Arch Pediatr Adolesc Med, 149 (7):786-791.

Kumarihamy SL, Subasinghe LD, Jayasekara P, Kularatna SM, Palipana PD. 2011. The prevalence of Early Childhood Caries in 1-2 yrs olds in a semi-urban area of Sri Lanka. BMC Res Notes, 4:336. 
Leal SC, Bronkhorst EM, Fan M, Frencken JE. 2012. Untreated cavitated dentine lesions: impact on children's quality of life. Caries Res, 46 (2):102-106.

Lee JY, Bouwens TJ, Savage MF, Vann WF, Jr. 2006. Examining the costeffectiveness of early dental visits. Pediatr Dent, 28 (2):102-105.

Li Y, Caufield PW, Dasanayake AP, Wiener HW, Vermund SH. 2005. Mode of delivery and other maternal factors influence the acquisition of Streptococcus mutans in infants. J Dent Res, 84 (9):806-811.

Livny A, Assali R, Sgan-Cohen HD. 2007. Early Childhood Caries among a Bedouin community residing in the eastern outskirts of Jerusalem. BMC Public Health, 7:167.

Loesche W. 1986. Role of Streptococcus mutans in human dental decay. Microbiol Rev, 50 (4):353-380.

Long CM, Quinonez RB, Beil HA, Close K, Myers LP, Vann WF, Jr., Rozier RG. 2012. Pediatricians' assessments of caries risk and need for a dental evaluation in preschool aged children. BMC Pediatr, 12:49.

Lulic-Dukic O, Juric H, Dukic W, Glavina D. 2001. Factors predisposing to early childhood caries (ECC) in children of pre-school age in the city of Zagreb, Croatia. Coll Antropol, 25 (1):297-302.

Lussi A, Kohler N, Zero D, Schaffner M, Megert B. 2000. A comparison of the erosive potential of different beverages in primary and permanent teeth using an in vitro model. Eur J Oral Sci, 108 (2):110-114.

Lutz ME. 1990. The effects of family structure and regular places of care on preventive health care for children. Health Values, 14 (1):38-45.

Marinho VC, Higgins JP, Logan S, Sheiham A. 2002. Fluoride varnishes for preventing dental caries in children and adolescents. Cochrane Database Syst Rev, (3):CD002279.

Marsh PD, Moter A, Devine DA. 2011. Dental plaque biofilms: communities, conflict and control. Periodontol 2000, 55 (1):16-35.

Marthaler TM, O'Mullane DM, Vrbic V. 1996. The prevalence of dental caries in Europe 1990-1995. ORCA Saturday afternoon symposium 1995. Caries Res, 30 (4):237-255.

Micheelis W, Schiffner U, Hoffmann T, Kerschbaum T, John M. 2007. Ausgewählte Ergebnisse der Deutschen Mundgesundheitsstudie (DMS IV). Dtsch Zahnärztl Z, 62:218-240. 
Mikkelsen L. 1996. Effect of sucrose intake on numbers of bacteria in plaque expressing extracellular carbohydrate metabolizing enzymes. Caries Res, 30 (1):65-70.

Milgrom P, Riedy CA, Weinstein P, Tanner AC, Manibusan L, Bruss J. 2000. Dental caries and its relationship to bacterial infection, hypoplasia, diet, and oral hygiene in 6- to 36-month-old children. Community Dent Oral Epidemiol, 28 (4):295-306.

Milnes AR. 1996. Description and epidemiology of nursing caries. J Public Health Dent, 56 (1):38-50.

Ministerium für Arbeit Soziales Gesundheit und Familie des Landes Brandenburg (MASGF). 2008. Gesunde Zähne für ein fröhliches Lächeln - Zur Mundgesundheit der Kinder und Jugendlichen im Land Brandenburg, Beiträge zur Gesundheits- und Sozialberichterstattung. Potsdam.

Mohebbi SZ, Virtanen JI, Vahid-Golpayegani M, Vehkalahti MM. 2008. Feeding habits as determinants of early childhood caries in a population where prolonged breastfeeding is the norm. Community Dent Oral Epidemiol, 36 (4):363-369.

Navarro-Rubio MD, Jovell AJ, Schor EL. 1995. Socioeconomic status and preventive health-care use by children in Spain. Am J Prev Med, 11 (4):256-262.

Neumann AS, Lee KJ, Gussy MG, Waters EB, Carlin JB, Riggs E, Kilpatrick NM. 2011. Impact of an oral health intervention on pre-school children $<3$ years of age in a rural setting in Australia. J Paediatr Child Health, 47 (6):367-372.

Nies SM, Schauß SS, Siahi-Benlarbi R, Schulz-Weidner N, Wetzel WE. 2008. Häufigkeit und Typisierung der Milchzahnkaries bei Kindergartenkindern in Mittelhessen. Oralprophylaxe \& Kinderzahnheilkunde, 30:106-111.

Niji R, Arita K, Abe Y, Lucas ME, Nishino M, Mitome M. 2010. Maternal age at birth and other risk factors in early childhood caries. Pediatr Dent, 32 (7):493-498.

Oliveira MJ, Paiva SM, Martins LH, Pordeus IA, Lima YB, Cury JA. 2006. Influence of rinsing and expectoration after toothbrushing on fluoride dose and ingested amount by use of conventional and children's fluoride dentifrices. Braz Dent J, $17(2): 100-105$.

Pieper K, Jablonski-Momeni A. 2008. Prävalenz der Milchzahnkaries in Deutschland. Oralprophylaxe \& Kinderzahnheilkunde, 30:6-10. 
Pitts NB, Boyles J, Nugent ZJ, Thomas N, Pine CM. 2005. The dental caries experience of 5-year-old children in England and Wales (2003/4) and in Scotland (2002/3). Surveys co-ordinated by the British Association for the Study of Community Dentistry. Community Dent Health, 22 (1):46-56.

Plutzer K, Spencer AJ. 2008. Efficacy of an oral health promotion intervention in the prevention of early childhood caries. Community Dent Oral Epidemiol, 36 (4):335-346.

Poorterman JH, Aartman IH, Kieft JA, Kalsbeek H. 2000. Value of bite-wing radiographs in a clinical epidemiological study and their effect on the DMFS index. Caries Res, 34 (2):159-163.

Poulsen S, Malling Pedersen M. 2002. Dental caries in Danish children: 1988-2001. Eur J Paediatr Dent, 3 (4):195-198.

Psoter WJ, Pendrys DG, Morse DE, Zhang H, Mayne ST. 2006. Associations of ethnicity/race and socioeconomic status with early childhood caries patterns. J Public Health Dent, 66 (1):23-29.

Public Health Service Commissioned Corps (PHSCC). 2000. Oral health in America: a report of the Surgeon General. J Calif Dent Assoc, 28 (9):685-695.

Qin M, Li J, Zhang S, Ma W. 2008. Risk factors for severe early childhood caries in children younger than 4 years old in Beijing, China. Pediatr Dent, 30 (2):122128.

Robke FJ, Buitkamp M. 2002. Häufigkeit der Nuckelflaschenkaries bei Vorschulkindern in einer westdeutschen Großstadt. Oralprophylaxe, 24:59- 65.

Robke FJ. 2008. Effects of nursing bottle misuse on oral health. Prevalence of caries, tooth malalignments and malocclusions in North-German preschool children. $J$ Orofac Orthop, 69 (1):5-19.

Rosenblatt A, Zarzar P. 2002. The prevalence of early childhood caries in 12- to 36month-old children in Recife, Brazil. ASDC J Dent Child, 69 (3):319-324.

Santos AP, Soviero VM. 2002. Caries prevalence and risk factors among children aged 0 to 36 months. Pesqui Odontol Bras, 16 (3):203-208.

Schäfer T, Schneider A, Hussein R, Schwartz FW. 2012. BARMER GEK Zahnreport 2012. Siegburg: BARMER GEK.

Schroth RJ, Cheba V. 2007. Determining the prevalence and risk factors for early childhood caries in a community dental health clinic. Pediatr Dent, 29 (5):387396. 
Schroth RJ, Levi J, Kliewer E, Friel J, Moffatt ME. 2013. Association between iron status, iron deficiency anaemia, and severe early childhood caries: a casecontrol study. BMC Pediatr, 13:22.

Senkel H, Heinrich-Weltzien R. 2008. Milchzahnkaries vor dem Hintergrund des generellen Kariesrückganges bei Kindern und Jugendlichen. Oralprophylaxe \& Kinderzahnheilkunde, 30:38-42.

Shaoul R, Gaitini L, Kharouba J, Darawshi G, Maor I, Somri M. 2012. The association of childhood iron deficiency anaemia with severe dental caries. Acta Paediatr, 101 (2):e76-79.

Simmer JP, Hu JC. 2001. Dental enamel formation and its impact on clinical dentistry. J Dent Educ, 65 (9):896-905.

Spitzenverband der landwirtschaftlichen Sozialversicherung (SVLFG). 2012.

Ausgaben der GKV für Gruppen- und Individualprophylaxe sowie

Kinderfrüherkennungs-Untersuchungen (FU) in den Jahren 2007 - 2011 http://www.daj.de/fileadmin/user_upload/PDF_Downloads/Ausgaben_GKV200 7bis2011.pdf.

Splieth $\mathrm{CH}$, Treuner A, Berndt C. 2009. Orale Gesundheit im Kleinkindalter. Prävention und Gesundheitsförderung, 4 (2):119-124.

Stecksen-Blicks C, Sunnegardh K, Borssen E. 2004. Caries experience and background factors in 4-year-old children: time trends 1967-2002. Caries Res, 38 (2):149-155.

Strippel H. 2002. Gesundheitsökonomie der Prävention, Beispiel Kariesprophylaxe. Die BKK, 11:477-481.

Subramaniam P, Prashanth P. 2012. Prevalence of early childhood caries in 8 - 48 month old preschool children of Bangalore city, South India. Contemp Clin Dent, 3 (1):15-21.

Sufia S, Khan AA, Chaudhry S. 2009. Maternal factors and child's dental health. J Oral Health Comm Dent, 3:45-48.

Tinanoff N, Kanellis MJ, Vargas CM. 2002. Current understanding of the epidemiology mechanisms, and prevention of dental caries in preschool children. Pediatr Dent, 24 (6):543-551.

Tsai Al, Chen CY, Li LA, Hsiang CL, Hsu KH. 2006. Risk indicators for early childhood caries in Taiwan. Community Dent Oral Epidemiol, 34 (6):437-445. 
Twetman S. 2008. Prevention of early childhood caries (ECC)--review of literature published 1998-2007. Eur Arch Paediatr Dent, 9 (1):12-18.

Vachirarojpisan T, Shinada K, Kawaguchi Y, Laungwechakan P, Somkote T, Detsomboonrat P. 2004. Early childhood caries in children aged 6-19 months. Community Dent Oral Epidemiol, 32 (2):133-142.

Wagner Y, Greiner S, Bischof T, Borgert G, Heinrich-Weltzien R. 2011. Einfluss der Wöchnerinnenberatung auf die Zahngesundheit von 5-jährigen Kindergartenkindern in Vorarlberg.

Wan AK, Seow WK, Walsh LJ, Bird P, Tudehope DL, Purdie DM. 2001. Association of Streptococcus mutans infection and oral developmental nodules in predentate infants. J Dent Res, 80 (10):1945-1948.

Warren JJ, Weber-Gasparoni K, Marshall TA, Drake DR, Dehkordi-Vakil F, Kolker JL, Dawson DV. 2008. Factors associated with dental caries experience in 1-yearold children. J Public Health Dent, 68 (2):70-75.

Weintraub JA, Ramos-Gomez F, Jue B, Shain S, Hoover Cl, Featherstone JD, Gansky SA. 2006. Fluoride varnish efficacy in preventing early childhood caries. J Dent Res, 85 (2):172-176.

Wetzel WE. 2008. Nach der Nuckel- die Rennfahrerflasche?. Dtsch Zahnärztl Z, $59: 55$

Willems S, Vanobbergen J, Martens L, De Maeseneer J. 2005. The independent impact of household- and neighborhood-based social determinants on early childhood caries: a cross-sectional study of inner-city children. Fam Community Health, 28 (2):168-175.

World Health Organization (WHO). 1997. Oral health surveys : basic methods. 4. Aufl. Geneva.

World Health Organization (WHO). 2003. Global strategy for infant and young child feeding. Geneva.

Wyne AH. 1999. Early childhood caries: nomenclature and case definition. Community Dent Oral Epidemiol, 27 (5):313-315.

Zhou Y, Yang JY, Lo EC, Lin HC. 2012. The contribution of life course determinants to early childhood caries: a 2-year cohort study. Caries Res, 46 (2):87-94. 


\section{Anhang}

Anhang 1: Liste der wissenschaftlichen Vorträge und Veröffentlichungen

Anhang 2: Anschreiben an die Eltern mit Einwilligung in die anonyme Auswertung der Fragebogen- und Mundgesundheitsdaten

Anhang 3: Elternfragebogen mit Angaben zum Mundgesundheitsverhalten und sozialem Status

Anhang 4: Lebenslauf

Anhang 5: Danksagung

Anhang 6: Ehrenwörtliche Erklärung 


\section{Liste der wissenschaftlichen Vorträge und Veröffentlichungen}

- $\quad$ „Frühkindliche Karies und assoziierte Risikofaktoren bei Kleinkindern im Land Brandenburg“,

Kongressbeitrag beim 62. wissenschaftlicher Kongress des Bundesverbandes der Ärztinnen und Ärzte des Öffentlichen Gesundheitsdienstes und des Bundesverbandes der Zahnärzte des Öffentlichen Gesundheitsdienstes, 10.12. Mai 2012 in Erfurt/ Thüringen, Gesundheitswesen 2012; 74 - V64

- $\quad$ „Frühkindliche Karies bei 0- bis 3-jährigen Kita-Kindern und Angaben der Eltern zum Gesundheitsverhalten im Land Brandenburg- Vorstellung erster Ergebnisse des Kooperationsprojektes“,

Darstellung der Ergebnisse des Kooperationsprojektes bei der Besprechung der Sachgebietsleiter des Ministeriums für Umwelt, Gesundheit und Verbraucherschutz des Landes Brandenburg, 22. Juni 2011 in Potsdam/ Brandenburg

- $\quad$ „Frühkindliche Karies und assoziierte Risikofaktoren bei Kleinkindern im Land Brandenburg" angemeldeter Kongressbeitrag zum Deutschen Zahnärztetag 2013, Frankfurt am Main, 8.- 9. November 2013

- $\quad$ Deichsel M, Rojas G, Lüdecke K, Heinrich-Weltzien R. 2012. Frühkindliche Karies und assoziierte Risikofaktoren bei Kleinkindern im Land Brandenburg. Bundesgesundheitsblatt, 55 (11-12): 1504-1511

Rojas G, Deichsel M, Lüdecke K, Heinrich-Weltzien R. 2013. Frühkindliche Karies bei Kleinkindern im Land Brandenburg - eine landesweite Studie zur Epidemiologie und zu Risikofaktoren. Zahnärztlicher Gesundheitsdienst, 1.13: 10 
- $\quad$ Rojas G, Deichsel M, Lüdecke K, Heinrich-Weltzien R. 2013. Erstmals Daten zu den jüngsten Kindern- Frühkindliche Karies bei Kleinkindern im Land Brandenburg- eine landesweite Studie zur Epidemiologie und zu Risikofaktoren. Zahnärzteblatt Brandenburg. 2.13: 14-15

- $\quad$ Rojas G, Deichsel M, Lüdecke K, Heinrich-Weltzien R. 2013. Ergebnisse einer landesweiten Studie im Land Brandenburg zur frühkindlichen Karies.

Deutsche Arbeitsgemeinschaft für Jugendzahnpflege e.V., http://daj.de/Ergebnisse-einer-landesweiten.102+M5ed820a595c.0.html, 13.03.2013

- $\quad$ Rojas G, Deichsel M, Lüdecke K, Heinrich-Weltzien R. 2013. Frühkindliche Karies bei Kleinkindern im Land Brandenburg- Eine landesweite Studie zur Epidemiologie und zu Risikofaktoren. Kooperationsverbund gesundheitliche Chancengleichheit., http://www.gesundheitlichechancengleichheit.de/koordinierungsstellen/fruehkindliche-karies-beikleinkindern-im-land-brandenburg/, 31.01.2013 


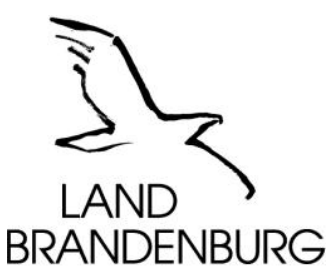

Ministerium für Arbeit, Soziales, Gesundheit und Familie

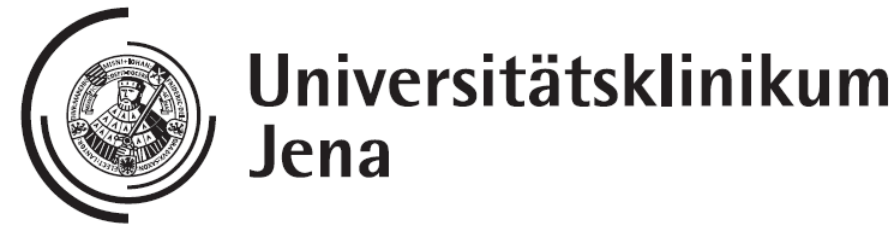

Medizinische Fakultät

Zentrum für Zahn-, Mund- und Kieferheilkunde Poliklinik für Präventive Zahnheilkunde Prof. Dr. Roswitha Heinrich-Weltzien (Leiterin) Bachstr. 18, D-07740 Jena

Telefon: $03641 \cdot 934803$

Telefax: $03641 \cdot 934802$

E-Mail: roswitha.heinrich-weltzien@med.uni-jena.de

Sehr geehrte Familie

ich heiße Martin Deichsel, studiere an der Universität Jena Zahnmedizin und arbeite an dem Kooperationsprojekt des Landesgesundheitsamtes Brandenburg und der Poliklinik für Präventive Zahnheilkunde des Universitätsklinikums Jena. In diesem Projekt sollen die Zusammenhänge zwischen der Mundgesundheit und den Zahnpflegegewohnheiten von Kleinkindern untersucht werden.

Um Eltern zukünftig beim gesunden Aufwachsen ihrer Kinder besser unterstützen zu können, gehört auch eine Elternbefragung zu diesem Projekt.

Bitte nehmen Sie sich einen Moment Zeit und beantworten Sie unsere Fragen, die anschließend anonym ausgewertet werden.

Für Ihre Mitarbeit bedanke ich mich im Namen der Poliklinik für Präventive Zahnheilkunde und des Landesgesundheitsamtes Brandenburg. Zehn Zahnärztliche Dienste im Land Brandenburg unterstützen die Organisation des Projektes und werden die beantworteten Fragebögen an das Universitätsklinikum Jena weiterleiten.

Bitte legen Sie den ausgefüllten Fragebogen und dieses Schreiben mit der unterschriebenen Einverständniserklärung in den Umschlag zurück und geben inn in der KITA ab. Dort nimmt inn das Team Ihres Zahnärztlichen Dienstes in Empfang.

Mit freundlichen Grüßen

Martin Deichsel

Ich bin damit einverstanden, dass die Daten meines Kindes

im Rahmen des Projektes „Frühkindliche Karies bei Kleinkindern und Angaben der Eltern zum Gesundheitsverhalten im Land Brandenburg" in der Poliklinik für Präventive Zahnheilkunde, des Universitätsklinikums Jena, anonymisiert gespeichert und ausgewertet werden.

Ja, ich bin einverstanden

Nein, ich bin nicht einverstanden

Datum:

Unterschrift 


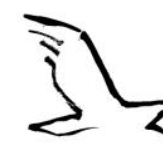

LAND

BRANDENBURG

Ministerium für Arbeit, Soziales,

Gesundheit und Familie

\section{Elternbefragung zum Gesundheitsverhalten}

Die Beantwortung der Fragen wird ungefähr 5 Minuten dauern. Ihre Angaben werden anonymisiert und dienen ausschließlich der erfolgreichen Umsetzung unseres Projektes. Vielen Dank für Ihre Unterstützung.

1) Wie alt ist Ihr Kind?
$\square \quad$ bis 12 Monate
13 - 24 Monate
25 - 36 Monate

2) Besitzt Ihr Kind eine eigene Zahnbürste?
$\square$ ja
$\square \quad$ nein

3) Wann haben Sie begonnen, Ihrem Kind die Zähne zu putzen?

$\square \quad$ seit Durchbruch des 1. Milchzahnes

$\square \quad$ im 1. Lebensjahr

$\square \quad$ im 2. Lebensjahr

$\square \quad$ noch gar nicht (bitte weiter mit Frage 8 )

4) Putzt sich Ihr Kind schon selbstständig die Zähne?

$\square \quad$ ja
$\square \quad$ nein

5) Wann putzen Sie Ihrem Kind bzw. putzt sich Ihr Kind die Zähne?

$\square$ morgens

$\square$ abends

$\square \quad$ morgens und abends

$\square \quad$ öfter

6) Falls sich Ihr Kind schon selbstständig die Zähne putzt; wie oft putzen Sie die Zähne Ihres Kindes nach?

$\begin{array}{ll}\square & \text { immer } \\ \square & \text { einmal täglich } \\ \square & \text { unregelmäßig } \\ \square & \text { nie }\end{array}$

7) Was für eine Zahnpasta benutzt Ihr Kind?

eine fluoridhaltige Kinderzahnpasta

$\square \quad$ eine fluoridfreie Kinderzahnpasta

$\square \quad$ eine Erwachsenenzahnpasta

$\square \quad$ weiß nicht 
8) Bekommt oder bekam Ihr Kind Fluoridtabletten (Fluoretten, Zymafluor, etc.)?

$\square$ ja, immer noch

$\square \quad$ ja, bis zum Lebensjahr

$\square$ nein

$\square \quad$ weiß nicht

9) Welches Speisesalz verwenden Sie im Haushalt?

$\square \quad$ Einfaches

$\square \quad$ mit Jod angereichertes

$\square \quad$ mit Jod und Fluorid angereichertes

10) Haben Sie Ihr Kind gestillt?

$\square$ nein

$\square$ ja

Wie lange wurde Ihr Kind gestillt?

$\square$ bis zum Lebensmonat

$\square \quad$ bis zum Durchbruch des 1. Milchzahnes

bis jetzt

11) Bekommt Ihr Kind die Flasche?

$\square \quad$ ja, bis jetzt ja, bis zum ___ Lebensmonat

$\square$ nein

12) Bekommt Ihr Kind nachts die Flasche?

$\square \quad$ ja
$\square \quad$ nein

13) Waren Sie mit Ihrem Kind schon beim Zahnarzt?

$\square \quad$ nein (bitte weiter mit Frage 16)

$\square$ ja

$\square \quad$ mit etwa 6 Monaten

口 nach dem 1. Geburtstag

口 nach dem 2. Geburtstag

14) Was war der Grund des ersten Zahnarztbesuches?

$\square \quad$ Kontrolle

$\square \quad$ Aufforderung durch den Zahnärztlichen Dienst in KiTa

口 Schmerzen

15) Welche Empfehlungen haben Sie dabei von Ihrem Zahnarzt erhalten?

- Aufklärung zur Nuckelflaschenkaries

$\square$ Verwendung einer fluoridhaltigen Kinderzahnpasta

- Nachputzen der Zähne durch die Eltern

- Ernährungsberatung

$\square \quad$ Verwendung von Speisesalz mit Fluorid

$\square \quad$ keine 
16) Wie oft erhält Ihr Kind zuckerreiche Getränke zwischen den Hauptmahlzeiten?

$\square$ selten

- bis zu 3 mal in der Woche

$\square \quad$ einmal täglich

2 - 3mal täglich

$\square \quad$ öfter

17) Bekommt Ihr Kind regelmäßig zuckerhaltige Medikamente?

(z.B. Husten- oder Antibiotikasaft)

$\square$ ja

$\square$ nein

․ weiß nicht

18) Wie alt sind Sie?

$\square$ bis 20 Jahre

$\square$ bis 25 Jahre

$\square$ bis 30 Jahre

$\square$ bis 35 Jahre

口 über 35 Jahre

19) Wie viele Kinder leben in Ihrem Haushalt?
$\square 1$
$\square \quad 2$
$\square 3$
4 und mehr

20) Wie viele Erwachsene leben in Ihrem Haushalt?

$\square \quad 1$
$\square \quad 2$

21) Angaben zum Schulabschluss

ohne Schulabschluss oder weniger als 10 Klassen

10. Klasse

mehr als 10 Klassen

22) Angaben zur Erwerbstätigkeit

nicht erwerbstätig

erwerbstätig (Vollzeit und Teilzeit)

Mutter Vater

Vielen Dank für Ihre Unterstützung.

Bitte legen Sie den ausgefüllten Fragebogen in den Umschlag und geben ihn in der KITA ab. 


\section{Lebenslauf}

\section{Persönliche Daten}

Name: $\quad$ Martin Deichsel

Anschrift: $\quad$ Luckenberger Str. 3, 14770 Brandenburg an der Havel

Geburtsdatum: $\quad$ 13. Juni 1987

Geburtsort: $\quad$ Brandenburg an der Havel

Staatsangehörigkeit: deutsch

\section{Ausbildung}

1993-1999

Luckenberger Grundschule, Brandenburg

1999-2006

von Saldern Gymnasium, Brandenburg

2003-2004

Santa Teresa High School, San Jose, CA, USA

06/ 2006

Abitur (Durchschnittsnote 1,3)

2006-2011

Zahnmedizinstudium an der FSU Jena

12/ 2011

Approbation als Zahnarzt (Staatsexamensnote „sehr gut")

\section{Berufstätigkeiten}

02/ 2012-dato

Weiterbildungsassistent zum Fachzahnarzt

"Allgemeine Zahnheilkunde" bei

ZA Dr. med. Erwin Deichsel, Brandenburg, 


\section{Danksagung}

Mein herzlicher Dank gilt meiner Doktormutter Frau Prof. Dr. med. habil. Roswitha Heinrich-Weltzien, Leiterin der Poliklinik für Präventive Zahnheilkunde und Kinderzahnheilkunde des Universitätsklinikums der Friedrich- Schiller- Universität Jena, für die Überlassung dieses hochinteressanten Themas sowie für die jahrelange unermüdliche Unterstützung und Ihren fachlichen Rat. Ohne Ihre fachkundige Begleitung der Arbeit wäre die Umsetzung in dieser Form nicht möglich gewesen.

Weiterhin möchte ich mich bei Frau Dr. med. Gudrun Rojas, Sachgebietsleiterin des Zahnärztlichen Dienstes der Stadt Brandenburg, für Ihre Hilfe in Wort und Tat, Ihre Ratschläge und Ihren ausdauernden Einsatz für das Kooperationsprojekt ganz besonders bedanken. Durch Ihr Engagement hat die Arbeit viele interessante Aspekte hinzugewonnen und Ihre Rolle als Vermittlerin zwischen den Partnern des Kooperationsprojektes hat die Abstimmung in vielen Bereichen erleichtert.

Ebenso bedanke ich mich bei Frau Karin Lüdecke, Mitarbeiterin des Landesamtes für Umwelt, Gesundheit und Verbraucherschutz, Abteilung Gesundheit, die mir freundlicherweise die Rohdaten der Mundgesundheitsuntersuchungen zur Verfügung gestellt hat und mir bei der Auswertung dieser, mit fachlichem Rat zur Seite stand sowie durch Ihre Anmerkungen die Fertigstellung der Arbeit vorangebracht hat.

Den Mitarbeiter/- innen des Landesamtes für Umwelt, Gesundheit und Verbraucherschutz sowie des Ministeriums für Umwelt, Gesundheit und Verbraucherschutz des Landes Brandenburg, danke ich für Ihre Unterstützung des Kooperationsprojektes, ohne die die Realisierung dieser Studie und Dissertationsschrift nicht möglich gewesen wäre.

Ein ganz besonderer Dank gilt den vielen Mitarbeiter/-innen und Zahnärztinnen der beteiligten Landkreise und kreisfreien Städte für Ihre Arbeit vor Ort in den Kindertagesstätten, für die Erhebung der Mundgesundheitsdaten und die Verteilung sowie das Zusenden der Fragebögen, die die Grundlage dieser Arbeit gebildet haben. 


\section{Ehrenwörtliche Erklärung}

Hiermit erkläre ich, dass mir die Promotionsordnung der Medizinischen Fakultät der Friedrich-Schiller-Universität bekannt ist,

ich die Dissertation selbst angefertigt habe und alle von mir benutzten Hilfsmittel, persönlichen Mitteilungen und Quellen in meiner Arbeit angegeben sind,

mich folgende Personen bei der Auswahl und Auswertung des Materials sowie bei der Herstellung des Manuskripts unterstützt haben:

Frau Prof. Dr. Roswitha Heinrich- Weltzien,

Frau Dr. Gudrun Rojas

Frau Karin Lüdecke

die Hilfe eines Promotionsberaters nicht in Anspruch genommen wurde und dass Dritte weder unmittelbar noch mittelbar geldwerte Leistungen von mir für Arbeiten erhalten haben, die im Zusammenhang mit dem Inhalt der vorgelegten Dissertation stehen,

dass ich die Dissertation noch nicht als Prüfungsarbeit für eine staatliche oder andere wissenschaftliche Prüfung eingereicht habe und

dass ich die gleiche, eine in wesentlichen Teilen ähnliche oder eine andere Abhandlung nicht bei einer anderen Hochschule als Dissertation eingereicht habe.

Ort, Datum

Unterschrift des Verfassers 